\title{
EXISTENCE OF A SOLUTION TO A NON-MONOTONE DYNAMIC MODEL IN POROPLASTICITY WITH MIXED BOUNDARY CONDITIONS
}

\author{
SEBASTIAn OWCZAREK
}

\begin{abstract}
In this note, we investigate a non-monotone and non-coercive dynamic model of poroplasticity with mixed boundary conditions. The existence of the solution to this non-monotone model, where the inelastic constitutive equation is satisfied in the sense of Young measures, is proved using the coercive and monotone approximations.
\end{abstract}

\section{Introduction}

This article presents mathematical existence results for a dynamic model describing an inelastic deformation process in porous materials when we apply external forces. Porous materials are granular and they are saturated by liquids (a good example of a porous material is clay). The dynamic system of equations

2010 Mathematics Subject Classification. 74C05, 35L15, 74H20.

Key words and phrases. Porous media, Biot model, inelastic deformation, Young measure, Yosida approximation, coercive approximation. 
of the theory of poroplasticity can be written in the form

$$
\begin{aligned}
\rho u_{t t}(x, t)-\operatorname{div}_{x} T(x, t)+\nabla_{x} p(x, t) & =F(x, t), \\
c \Delta_{x} p(x, t)-\operatorname{div}_{x} u_{t}(x, t) & =f(x, t), \\
T(x, t) & =\mathcal{D}\left(\varepsilon(x, t)-\varepsilon^{p}(x, t)\right), \\
\varepsilon(x, t) & =\frac{1}{2}\left(\nabla_{x} u(x, t)+\nabla_{x}^{T} u(x, t)\right), \\
\varepsilon_{t}^{p}(x, t) & =\mathcal{F}(Y(T(x, t))) \frac{\partial P}{\partial T}(T(x, t)),
\end{aligned}
$$

where $x \in \Omega$ and $\Omega$ is a bounded and open set in $\mathbb{R}^{3}$ with smooth boundary $\partial \Omega$. Moreover, $t>0$ represents time. Notice that the system of equations (1.1) consists of linear partial differential equations and ordinary differential equations for internal variables. The linear partial differential equations in (1.1) are called the Biot model - for more information we refer to [16], [19]. The last equation in (1.1) is called the constitutive equation. This constitutive equation, which is often used in practice, was introduced by W. Ehlers in the work [12]. The set of internal variables proposed by W. Ehlers consists of nine components of the plastic strain $\varepsilon^{p}$ only (for the physical understanding of the constitutive equations we also refer to [12]). $\varepsilon$ is the infinitesimal strain tensor.

In the system (1.1) the unknowns are the displacement $u: \Omega \times[0, T] \rightarrow \mathbb{R}^{3}$, the pressure of the fluid $p: \Omega \times[0, T] \rightarrow \mathbb{R}$, the Cauchy stress tensor $T: \Omega \times[0, T] \rightarrow \mathcal{S}^{3}$ and the plastic strain tensor $\varepsilon^{p}: \Omega \times[0, T] \rightarrow \mathcal{S}^{3}\left(\mathcal{S}^{3}\right.$ denotes the set of symmetric $3 \times 3$-matrices). $F: \Omega \times[0, T] \rightarrow \mathbb{R}^{3}$ and $f: \Omega \times[0, T] \rightarrow \mathbb{R}$ are given functions. $\mathcal{D}: \mathcal{S}^{3} \rightarrow \mathcal{S}^{3}$ is a linear, symmetric, positive definite operator (elasticity tensor), which is assumed to be constant in time and space. $\rho>0$ is the mass density which we assume to be constant and $c>0$ is also a constant.

The functions $Y: \mathcal{S}^{3} \rightarrow \mathbb{R}$ and $P: \mathcal{S}^{3} \rightarrow \mathbb{R}$ are given functions. We assume that they are convex homogeneous polynomials of the same growth, $\mathcal{F} \in$ $C^{1}\left(\mathbb{R} ; \mathbb{R}_{+}\right)$and $\mathcal{F}$ is a monotone function with polynomial growth, which means that there exist $\alpha>1$ and constants $m, M>0$ such that

$$
m|s|^{\alpha} \leq \mathcal{F}(s) \leq M|s|^{\alpha} \quad \text { for large }|s|
$$

We also assume, that $Y(0)=0$ and $\mathcal{F}(0)=0$.

The system (1.1) is considered with mixed boundary conditions

$$
\begin{aligned}
u(x, t)=g_{d}(x, t) & \text { for } x \in \Gamma_{d} \text { and } t \geq 0 \\
(T(x, t)-p(x, t) I) n(x)=g_{n}(x, t) & \text { for } x \in \Gamma_{n} \text { and } t \geq 0, \\
p(x, t)=g_{p}(x, t) & \text { for } x \in \Gamma_{p} \text { and } t \geq 0 \\
c \frac{\partial p}{\partial n}(x, t)=g_{v}(x, t) & \text { for } x \in \Gamma_{v} \text { and } t \geq 0
\end{aligned}
$$


where $n(x)$ is the exterior unit normal vector to the boundary $\partial \Omega$ at the point $x$, $\Gamma_{d}, \Gamma_{n}, \Gamma_{p}, \Gamma_{v}$ are open subsets of $\partial \Omega$ satisfying $\partial \Omega=\bar{\Gamma}_{d} \cup \bar{\Gamma}_{n}=\bar{\Gamma}_{p} \cup \bar{\Gamma}_{v}$, $\Gamma_{d} \cap \Gamma_{n}=\Gamma_{p} \cap \Gamma_{v}=\emptyset$ and $\mathcal{H}_{2}\left(\Gamma_{d}\right)>0, \mathcal{H}_{2}\left(\Gamma_{p}\right)>0$, where $\mathcal{H}_{2}$ denotes the 2-dimensional Hausdorff measure.

Finally, the system (1.1) is considered with initial conditions

$$
u(x, 0)=u^{0}(x), \quad u_{t}(x, 0)=u^{1}(x), \quad \varepsilon^{p}(x, 0)=\varepsilon^{p, 0}(x) .
$$

We say that the initial data $\left(u^{0}, u^{1}, \varepsilon^{p, 0}\right)$ satisfy the compatibility condition if

$$
u^{0}(x)=g_{d}(x, 0) \quad \text { and } \quad u^{1}(x)=\partial_{t} g_{d}(x, 0) \quad \text { for } x \in \Gamma_{d}
$$

and, for $x \in \Gamma_{n}$,

$$
\left(\mathcal{D}\left(\frac{1}{2}\left(\nabla_{x} u^{0}(x)+\nabla_{x}^{T} u^{0}(x)\right)-\varepsilon^{p, 0}(x)\right)-I p(x, 0)\right) \cdot n=g_{n}(x, 0),
$$

where $p(x, 0)$ is the solution of the equation

$$
c \Delta_{x} p(x, 0)-\operatorname{div}_{x} u_{t}(x, 0)=f(x, 0)
$$

with boundary conditions

$$
\begin{aligned}
p(x, 0) & =g_{p}(x, 0) & & \text { for } x \in \Gamma_{p}, \\
c \frac{\partial p}{\partial n}(x, 0) & =g_{v}(x, 0) & & \text { for } x \in \Gamma_{v} .
\end{aligned}
$$

REMARK 1.2. Notice that if the given data $f(0), g_{p}(0), g_{v}(0)$ and $u^{1}$ have the regularity: $f(0) \in L^{2}(\Omega ; \mathbb{R}), g_{p}(0), g_{v}(0) \in H^{1 / 2}(\Omega ; \mathbb{R})$ and $u^{1} \in H^{1}\left(\Omega ; \mathbb{R}^{3}\right)$, then the problem (1.6) with boundary conditions (1.7) has unique solution $p(0) \in$ $H^{1}(\Omega ; \mathbb{R})$ and the compatibility condition (1.5) makes sense.

Observe that the assumption on the functions $Y$ and $P$ implies that the constitutive equation in (1.1) is a nonlinear ordinary differential equation and the vector field

$$
N(T)=\mathcal{F}(Y(T)) \frac{\partial P}{\partial T}(T)
$$

is not monotone. It is easy to check that the function $N(T)$ satisfies the dissipative inequality: $-\rho \nabla_{\varepsilon^{p}} \psi\left(\varepsilon, \varepsilon^{p}\right) \cdot N(T) \geq 0$ (for the definition we refer to [2]), where $\rho \psi$ is the free energy function associated with the system (1.1) and it is given by the formula

$$
\rho \psi\left(\varepsilon, \varepsilon^{p}\right)=\frac{1}{2} \mathcal{D}\left(\varepsilon-\varepsilon^{p}\right)\left(\varepsilon-\varepsilon^{p}\right)=\frac{1}{2} T \mathcal{D}^{-1} T .
$$

The total free energy is of the form:

$$
\mathcal{E}\left(u_{t}, \varepsilon, \varepsilon^{p}\right)(t)=\frac{1}{2} \int_{\Omega} \rho\left|u_{t}(x, t)\right|^{2} d x+\int_{\Omega} \rho \psi\left(\varepsilon(x, t), \varepsilon^{p}(x, t)\right) d x .
$$


The considered model is non-coercive and non-monotone, because the quadratic form $\psi$ in (1.8) is only semi-positive definite and the constitutive function $N: \mathcal{S}^{3} \rightarrow$ $\mathcal{S}^{3}$ is not monotone (for the definitions, we refer to [2]).

The theory of inelastic deformation of metals is quite well examined if the considered model is monotone, which means that the nonlinearities in this model are monotone functions. This theory was developed by R. Temam, H.-D. Alber, K. Chełmiński and P. Gwiazda in the works [20], [3], [9], [10]. The theory of inelastic deformation for porous materials is not very well examined, because the constitutive function, proposed by W. Ehlers, is not monotone. In the literature there are not many mathematical results for this non-monotone model of poroplasticity (the monotone model describing the diffusion in porous materials was considered in [17]). The first work, where the non-monotone model of poroplasticity was studied, is [18]. The non-monotone quasi-static model in poroplasticity with only Dirichlet boundary conditions was considered. The main results is to pass to the limit in the monotone approximation which was proposed by author in [18]. This monotone approximation will be also used to the dynamic system (1.1). Passage to the limit with the monotone approximation is the main results of the present paper. In [18] the author defined a new notion of a solution for the quasi-static model in poroplasticity. This notion is weaker than the weak-type solution introduced in [3]. In the present paper we also have to define a weakertype solution than in [3]. This definition will be similar to the definition from [18] because the system (1.1) is also non-monotone and for the same reason as in [18] we can not obtain the standard weak-type solution. In this article we obtain a better regularity for time derivatives of the monotone approximate solution of the system (1.1) than in [18] and in the proof of this regularity we will not use the structure of the approximate constitutive function (as was done in [18]). The structure of the dynamic system allows us to consider the system (1.1) with mixed boundary conditions.

\section{Main theorem}

Assume that our data $F, f, g_{d}, g_{n}, g_{p} g_{v}, u^{0}, u^{1}, \varepsilon^{p, 0}$ have the following regularity

$$
\begin{gathered}
F \in H^{1}\left([0, T] ; L^{2}\left(\Omega ; \mathbb{R}^{3}\right)\right), \quad f \in H^{1}\left([0, T] ; L^{2}(\Omega ; \mathbb{R})\right), \\
g_{d} \in W^{3, \infty}\left([0, T] ; H^{1 / 2}\left(\Gamma_{d} ; \mathbb{R}^{3}\right)\right), \quad g_{n} \in W^{2, \infty}\left([0, T] ; H^{-1 / 2}\left(\Gamma_{n} ; \mathbb{R}^{3}\right)\right), \\
g_{p} \in W^{2, \infty}\left([0, T] ; H^{3 / 2}\left(\Gamma_{p} ; \mathbb{R}\right)\right), \quad g_{v} \in W^{2, \infty}\left([0, T] ; H^{1 / 2}\left(\Gamma_{v} ; \mathbb{R}\right)\right), \\
u^{0} \in H^{1}\left(\Omega ; \mathbb{R}^{3}\right), \quad u^{1} \in H^{1}\left(\Omega ; \mathbb{R}^{3}\right), \quad \varepsilon^{p, 0} \in L^{2}\left(\Omega ; \mathcal{S}^{3}\right), \\
\operatorname{div}\left(\varepsilon\left(u^{0}\right)-\varepsilon^{p, 0}\right) \in L^{2}\left(\Omega ; \mathbb{R}^{3}\right) .
\end{gathered}
$$


Before we formulate the definition of the solution to the problem (1.1) let us introduce spaces $V=\left\{v \in H^{1}\left(\Omega ; \mathbb{R}^{3}\right): v=0\right.$ on $\left.\Gamma_{d}\right\}$ and $W=\left\{w \in H^{1}(\Omega ; \mathbb{R})\right.$ : $w=0$ on $\left.\Gamma_{p}\right\}$.

Definition 2.1. Suppose that the given data satisfy $(2.1)-(2.5)$. We say that for $\beta>1$ a vector $u \in L^{\infty}\left(0, T ; W^{1,1+1 / \beta}\left(\Omega ; \mathbb{R}^{3}\right)\right)$ such that $u_{t} \in L^{\infty}(0, T$; $\left.L^{2}\left(\Omega ; \mathbb{R}^{3}\right)\right)$ and $u_{t t} \in L^{2}\left(0, T ;\left(H^{1}\left(\Omega ; \mathbb{R}^{3}\right)\right)^{*}\right)$, the function $p \in L^{2}\left(0, T ; H^{1}(\Omega ; \mathbb{R})\right)$, the inelastic deformation tensor $\varepsilon^{p} \in L^{\infty}\left(0, T ; L^{1+1 / \beta}\left(\Omega ; \mathcal{S}^{3}\right)\right)$ such that $\varepsilon_{t}^{p} \in$ $L^{1+1 / \beta}\left(0, T ; L^{1+1 / \beta}\left(\Omega ; \mathcal{S}^{3}\right)\right)$ and the Cauchy stress tensor $T \in L^{\infty}\left(0, T ; L^{2}\left(\Omega ; \mathcal{S}^{3}\right)\right)$ are solutions of the problem (1.1)-(1.3) if

(a) the functions $u$ and $p$ are in the form

$$
u(x, t)=v(x, t)+w(x, t), \quad p(x, t)=\widetilde{p}(x, t)+\widetilde{w}(x, t),
$$

where $w \in W^{3, \infty}\left(0, T ; H^{1}\left(\Omega ; \mathbb{R}^{3}\right)\right)$ and $\widetilde{w} \in W^{2, \infty}\left(0, T ; H^{2}(\Omega ; \mathbb{R})\right)$ are such functions that $\left.w\right|_{\Gamma_{d}}=g_{d}$ and $\left.\widetilde{w}\right|_{\Gamma_{p}}=g_{p}$. Functions $v$ and $\widetilde{p}$ satisfy the following system of the equations:

$$
\begin{aligned}
&\left\langle\rho v_{t t}, \bar{v}\right\rangle+ \int_{\Omega} \mathcal{D}\left(\varepsilon(v)-\varepsilon^{p}\right) \varepsilon(\bar{v}) d x-\int_{\Omega} p \operatorname{div} \bar{v} d x \\
&-\int_{\Omega} F \bar{v} d x-\int_{\Omega}\left(\mathcal{D}(\varepsilon(w)) \varepsilon(\bar{v})+\rho w_{t t}\right) \varepsilon(\bar{v}) d x+\int_{\Gamma_{n}} g_{n} \bar{v} d S(x), \\
& \int_{\Omega \times[0, T]} c \nabla \tilde{p} \nabla \phi d x d t-\int_{\Omega \times[0, T]} \operatorname{div} u \phi_{t} d x d t+\int_{\Omega} \operatorname{div} u^{0} \phi(0) d x \\
&=-\int_{\Omega \times[0, T]} f \phi d x d t-\int_{\Omega \times[0, T]} \nabla \widetilde{w} \nabla \phi d x d t+\int_{\Gamma_{v} \times[0, T]} g_{v} \phi d S(x),
\end{aligned}
$$

where the first equation is satisfied for all $\bar{v} \in V$ and for almost all $t \in(0, T)$, the second equation is satisfied for all $\phi \in C_{0}^{\infty}([0, T) ; W)$.

(b) The fifth equation in (1.1) is satisfied in the sense of Young measures i.e.

$$
\varepsilon_{t}^{p}(x, t)=\int_{\mathcal{S}^{3}} \mathcal{F}(Y(S)) \frac{\partial P}{\partial T}(S) d \nu_{(x, t)}(S),
$$

where $\nu_{(x, t)}$ is a Young measure satisfying

$$
T(x, t)=\int_{\mathcal{S}^{3}} S d \nu_{(x, t)}(S) \text { a.e. in } \Omega \times(0, T) .
$$

(c) $\varepsilon^{p}(x, 0)=\varepsilon^{p, 0}(x), u(0)=u^{0}(x), u_{t}(0)=u^{1}(x)$.

REMARK 2.2. In nonlinear problems with non-monotone nonlinearities many authors define weak solutions in measure sense only. For example we refer to [11], [13]-[15].

The next theorem is the main result of the present article. 
TheOREM 2.3 (Main Theorem). Suppose that the given data have the regularity required in (2.1)-(2.5) and assume that there exists a function $p^{*} \in$ $W^{2, \infty}\left(0, T ; H^{2}(\Omega ; \mathbb{R})\right)$ such that $\left.p^{*}\right|_{\Gamma_{p}}=g_{\left.p\right|_{\Gamma_{p}}}$. Let the functions $g_{d}$ and $g_{n}$ satisfy the weak-safe load condition (Definition 5.1) and the initial data satisfy the compatibility condition. Then, for all $T>0$ the system (1.1) with the boundary condition (1.2) and the initial condition (1.3) possesses a solution in the sense of Definition 2.1.

The proof of Theorem 2.3 is divided into two sections. In Section 4 we approximate the system (1.1) by coercive and almost monotone systems and we present the theory of the existence and uniqueness of solutions for each approximation step. Next (in Section 5), we pass to the limit with approximation and obtain the solutions in the sense of Definition 2.1.

In the literature, there exist many works on the existence of solutions to the dynamic Biot model. These solutions are not regular enough to prove existence and uniqueness of solutions for the approximation of the system (1.1). Before we formulate an approximation to the system (1.1), we present shortly the proof of existence and uniqueness of the solution to the dynamic Biot model with mixed boundary conditions. Better regularity for the time derivative of the displacement is the main result of the next section.

\section{Dynamic Biot model}

This section is devoted to studying of existence and uniqueness of the solution to the dynamic Biot model

$$
\begin{aligned}
\rho u_{t t}(x, t)-\operatorname{div}_{x} T(x, t)+\nabla_{x} p(x, t) & =F(x, t), \\
c \Delta_{x} p(x, t)-\operatorname{div}_{x} u_{t}(x, t) & =f(x, t), \\
T(x, t) & =\mathcal{D}(\varepsilon(u(x, t))), \\
\varepsilon(u(x, t)) & =\frac{1}{2}\left(\nabla_{x} u(x, t)+\nabla_{x}^{T} u(x, t)\right),
\end{aligned}
$$

with mixed boundary conditions

$$
\begin{aligned}
u(x, t) & =0 & & \text { for } x \in \Gamma_{d} \text { and } t \geq 0, \\
(T(x, t)-p(x, t) I) n(x, t) & =g_{n}(x, t) & & \text { for } x \in \Gamma_{n} \text { and } t \geq 0, \\
p(x, t) & =0 & & \text { for } x \in \Gamma_{p} \text { and } t \geq 0, \\
c \frac{\partial p}{\partial n}(x, t) & =g_{v}(x, t) & & \text { for } x \in \Gamma_{v} \text { and } t \geq 0
\end{aligned}
$$

and with initial conditions

$$
u(x, 0)=u^{0}(x), \quad u_{t}(x, 0)=u^{1}(x) .
$$

REMARK 3.1. Notice that the above model is linear and so it can be considered with homogeneous Dirichlet boundary conditions. 
Let us recall that $V=\left\{v \in H^{1}\left(\Omega ; \mathbb{R}^{3}\right): v=0\right.$ on $\left.\Gamma_{d}\right\}$ and $W=\{w \in$ $H^{1}(\Omega ; \mathbb{R}): w=0$ on $\left.\Gamma_{p}\right\}$.

Definition 3.2. We say that a vector $u \in L^{\infty}(0, T ; V)$ and a function $p \in$ $H^{1}(0, T ; W)$ such that $u_{t} \in L^{\infty}(0, T ; V)$ and $u_{t t} \in L^{\infty}\left(0, T ; L^{2}\left(\Omega ; \mathbb{R}^{3}\right)\right)$ are weak solutions of the problem (3.1)-(3.3) if for almost all $t \in[0, T]$

$$
\int_{\Omega} \rho u_{t t} v d x+\int_{\Omega} \mathcal{D}(\varepsilon(u)) \varepsilon(v) d x-\int_{\Omega} p \operatorname{div} v d x=\int_{\Omega} F v d x+\int_{\Gamma_{n}} g_{n} v d S(x)
$$

for all $v \in V$,

$$
\int_{\Omega} c \nabla p \nabla w d x+\int_{\Omega} \operatorname{div} u_{t} w d x=-\int_{\Omega} f w d x+\int_{\Gamma_{v}} g_{v} w d S(x)
$$

for all $w \in W$, and moreover, $u(x, 0)=u^{0}(x)$ and $u_{t}(x, 0)=u^{1}(x)$.

The following theorem is the main theorem of this section.

Theorem 3.3. Suppose that the external forces $F, f$ and boundary data $g_{n}$, $g_{v}$ satisfy:

$$
\begin{aligned}
F & \in H^{1}\left([0, T] ; L^{2}\left(\Omega ; \mathbb{R}^{3}\right)\right), & f \in H^{1}\left([0, T] ; L^{2}(\Omega ; \mathbb{R})\right) \\
g_{n} & \in W^{2, \infty}\left([0, T] ; H^{-1 / 2}\left(\Gamma_{n} ; \mathbb{R}^{3}\right)\right), & g_{v} \in H^{1}\left([0, T] ; H^{1 / 2}\left(\Gamma_{v} ; \mathbb{R}\right)\right)
\end{aligned}
$$

for all $T>0$. Moreover, assume that

$$
u^{0} \in H^{1}\left(\Omega ; \mathbb{R}^{3}\right), \quad u^{1} \in H^{1}\left(\Omega ; \mathbb{R}^{3}\right), \quad \operatorname{div}\left(\varepsilon\left(u^{0}\right)\right) \in L^{2}\left(\Omega ; \mathbb{R}^{3}\right)
$$

and that the initial data satisfy the compatibility conditions with boundary data. Then for all $T>0$ the system (3.1) with initial-boundary conditions (3.2) and (3.3) possesses a unique weak solution.

Proof. The proof of this theorem is divided into five steps.

Step 1. Preparation for the Galerkin method

The subset $V \subset H^{1}\left(\Omega ; \mathbb{R}^{3}\right)$ is the separable set. Then, there exists a sequence $\left\{v_{k}\right\}_{k=1}^{\infty}$ which is a basis of $V$. Using the Gram-Schmidt process we obtain that $\left\{v_{k}\right\}_{k=1}^{\infty}$ is an orthonormal basis of $L^{2}\left(\Omega ; \mathbb{R}^{3}\right)$ and $v_{k}=v_{k}(x)$, where $v_{k}$ is smooth inside $\Omega$ for all $k=1,2, \ldots$ (the Gram-Schmidt process does not impair the regularity). From similar argument there exists a sequence $\left\{w_{k}\right\}_{k=1}^{\infty}$ which is any basis of $W$ such that $w_{k}=w_{k}(x)$ and $w_{k}$ is smooth inside $\Omega$ for all $k=1,2, \ldots$ Let $V_{m}=\operatorname{span}\left\{v_{1}, \ldots, v_{m}\right\}$ and $W_{m}=\operatorname{span}\left\{w_{1}, \ldots, w_{m}\right\}$. We will find functions $u_{m}:[0, T] \rightarrow V_{m}$ and $p_{m}:[0, T] \rightarrow W_{m}$ in the form

$$
\begin{aligned}
& u_{m}(t)=\sum_{k=1}^{m} g_{m}^{k}(t) v_{k}, \\
& p_{m}(t)=\sum_{k=1}^{m} \widetilde{g}_{m}^{k}(t) w_{k}
\end{aligned}
$$


such that

$$
\begin{aligned}
\rho \int_{\Omega} u_{m}^{\prime \prime}(t) v_{k} d x+\int_{\Omega} \mathcal{D}\left(\varepsilon\left(u_{m}(t)\right)\right) \varepsilon\left(v_{k}\right) & d x-\int_{\Omega} p_{m}(t) \operatorname{div} v_{k} d x \\
& =\int_{\Omega} F v_{k} d x+\int_{\Gamma_{n}} g_{n} v_{k} d S(x)
\end{aligned}
$$

$$
\int_{\Omega} c \nabla p_{m}(t) \nabla w_{k} d x+\int_{\Omega} \operatorname{div} u_{m}^{\prime}(t) w_{k} d x=-\int_{\Omega} f w_{k} d x+\int_{\Gamma_{v}} g_{v} w_{k} d S(x)
$$

for all $k=1, \ldots, m$ and $0 \leq t \leq T$, where $u_{m}^{\prime}$ denotes the partial derivative of $u_{m}$ with respect to $t$.

We choose $u_{m}(0), u_{m}^{\prime}(0) \in V_{m}$ such that

$$
\begin{aligned}
u_{m}(0) & \rightarrow u^{0} & & \text { in } H^{1}\left(\Omega ; \mathbb{R}^{3}\right), \\
u_{m}^{\prime}(0) & \rightarrow u^{1} & & \text { in } H^{1}\left(\Omega ; \mathbb{R}^{3}\right), \\
\operatorname{div}\left(\varepsilon\left(u_{m}(0)\right)\right) & \rightarrow \operatorname{div}\left(\varepsilon\left(u^{0}\right)\right) & & \text { in } L^{2}\left(\Omega ; \mathbb{R}^{3}\right)
\end{aligned}
$$

as $m \rightarrow \infty$.

Step 2. Existence for each Galerkin approximation step

THEOREM 3.4. Let us suppose that our data have the regularity required in (3.4)-(3.6). Then for all natural $m=1,2, \ldots$ there exist unique functions $u_{m}(t)$ and $p_{m}(t)$ of the form (3.7) and (3.8) respectively, which satisfy (3.9) and (3.10).

Proof of the Theorem 3.4 immediately follows from the theory of ordinary differential equations and assumptions on the basis of $V$. The details are left to the reader.

Step 3. Energy estimates.

This step is the main part in the proof of Theorem 3.3.

THEOREM 3.5. Assume that our data have regularity required in (3.4)-(3.6). Then for all $T>0$ and $t \leq T$ there are positive constants $C(T), \widetilde{C}(T)$, not depending on $m$, such that

$$
\begin{gathered}
\left\|u_{m}^{\prime}(t)\right\|_{L^{2}\left(\Omega ; \mathbb{R}^{3}\right)}^{2}+\left\|u_{m}(t)\right\|_{H^{1}\left(\Omega ; \mathbb{R}^{3}\right)}^{2}+c \int_{0}^{t}\left\|p_{m}(\tau)\right\|_{H^{1}(\Omega ; \mathbb{R})}^{2} d \tau \leq C(T), \\
\left\|u_{m}^{\prime \prime}(t)\right\|_{L^{2}\left(\Omega ; \mathbb{R}^{3}\right)}^{2}+\left\|u_{m}^{\prime}(t)\right\|_{H^{1}\left(\Omega ; \mathbb{R}^{3}\right)}^{2}+\int_{0}^{t}\left\|p_{m}^{\prime}(\tau)\right\|_{H^{1}(\Omega ; \mathbb{R})}^{2} d \tau \leq \widetilde{C}(T) .
\end{gathered}
$$

Proof of Theorem 3.5. Note that it is enough to prove the inequality (3.12). Fix $T>0$. 
Calculating the time derivative of equations (3.9), (3.10), multiplying (3.9) by $\partial_{t}^{2}\left(g_{m}^{k}\right),(3.10)$ by $\partial_{t}\left(\widetilde{g}_{m}^{k}\right)$, summing both over $k=1, \ldots, m$, adding these results and integrating with respect to time we obtain

$$
\begin{aligned}
\frac{\rho}{2}\left\|u_{m}^{\prime \prime}(t)\right\|_{L^{2}\left(\Omega ; \mathbb{R}^{3}\right)}^{2} & +\frac{1}{2} \int_{\Omega} \mathcal{D}\left(\varepsilon\left(u_{m}^{\prime}(t)\right)\right) \varepsilon\left(u_{m}^{\prime}(t)\right) d x \\
& +c \int_{0}^{t}\left\|\nabla p_{m}^{\prime}(\tau)\right\|_{L^{2}\left(\Omega ; \mathbb{R}^{3}\right)}^{2} d \tau \\
= & \frac{\rho}{2}\left\|u_{m}^{\prime \prime}(0)\right\|_{L^{2}\left(\Omega ; \mathbb{R}^{3}\right)}^{2}+\frac{1}{2} \int_{\Omega} \mathcal{D}\left(\varepsilon\left(u_{m}^{\prime}(0)\right) \varepsilon\left(u_{m}^{\prime}(0)\right) d x\right. \\
& +\int_{0}^{t} \int_{\Omega} F^{\prime} u_{m}^{\prime \prime}(\tau) d x d \tau+\int_{0}^{t} \int_{\Gamma_{n}} g_{n}^{\prime} u_{m}^{\prime \prime}(\tau) d S(x) d \tau \\
& -\int_{0}^{t} \int_{\Omega} f^{\prime} p_{m}^{\prime}(\tau) d x d \tau+\int_{0}^{t} \int_{\Gamma_{v}} g_{v}^{\prime} p_{m}^{\prime}(\tau) d S(x) d \tau
\end{aligned}
$$

Let us estimate the first term on the right-hand side of (3.13). Observe that, from (3.10) we have that the following equation

$$
\begin{aligned}
\int_{\Omega} c \nabla p_{m}(0) \nabla w_{k} d x+\int_{\Omega} \operatorname{div} & u_{m}^{\prime}(0) w_{k} d x \\
& =-\int_{\Omega} f(0) w_{k} d x+\int_{\Gamma_{v}} g_{v}(0) w_{k} d S(x)
\end{aligned}
$$

is satisfied for any natural number $k=1, \ldots, m$. Multiplying equation (3.14) by $\widetilde{g}_{m}^{k}(0)$ and summing over $k=1, \ldots, m$ we obtain

$$
\begin{aligned}
c \int_{\Omega}\left|\nabla p_{m}(0)\right|^{2} d x+\int_{\Omega} \operatorname{div} u_{m}^{\prime}(0) p_{m}(0) d x & \\
& =-\int_{\Omega} f(0) p_{m}(0) d x+\int_{\Gamma_{v}} g_{v}(0) p_{m}(0) d S(x) .
\end{aligned}
$$

From the assumptions on the given initial data, Cauchy inequality and the trace theorem (which means that there exist a linear continuous mappings from $H^{1}(\Omega ; \mathbb{R})$ into $H^{1 / 2}(\partial \Omega ; \mathbb{R})$, see [1]) we obtain that $\left\|\nabla p_{m}(0)\right\|_{L^{2}\left(\Omega ; \mathbb{R}^{3}\right)}$ is bounded independently of $m$. Moreover, taking the equation (3.9) with $t=0$, multiplying by $g_{m}^{\prime \prime}(0)$ and summing over $k=1, \ldots, m$ we have

$$
\begin{aligned}
&\left\|\rho u_{m}^{\prime \prime}(0)\right\|_{L^{2}\left(\Omega ; \mathbb{R}^{3}\right)}^{2}+\int_{\Omega} \mathcal{D}\left(\varepsilon\left(u_{m}(0)\right)\right) \varepsilon\left(u_{m}^{\prime \prime}(0)\right) d x-\int_{\Omega} p_{m}(0) \operatorname{div} u_{m}^{\prime \prime}(0) d x \\
&=\int_{\Omega} F(0) u_{m}^{\prime \prime}(0) d x+\int_{\Gamma_{n}} g_{n}(0) u_{m}^{\prime \prime}(0) d S(x)
\end{aligned}
$$


Integrating by parts in the second and third term on the left-hand side of (3.15) we obtain

$$
\begin{aligned}
\left\|\rho u_{m}^{\prime \prime}(0)\right\|_{L^{2}\left(\Omega ; \mathbb{R}^{3}\right)}^{2}=\int_{\Omega} \operatorname{div} & \mathcal{D}\left(\varepsilon\left(u_{m}(0)\right)\right) u_{m}^{\prime \prime}(0) d x \\
& \quad-\int_{\Omega} \nabla p_{m}(0) u_{m}^{\prime \prime}(0) d x+\int_{\Omega} F(0) u_{m}^{\prime \prime}(0) d x .
\end{aligned}
$$

Using weighted Cauchy inequality in (3.16) and the regularity assumptions on the initial data we get that the first term on the right hand side of (3.13) is bounded independently of $m$.

The second term on the right-hand side of (3.13) is bounded, because $u_{m}^{\prime}(0) \rightarrow$ $u^{1}$ in $H^{1}\left(\Omega ; \mathbb{R}^{3}\right)$. Integrating by parts in the fourth integral on the right-hand side of (3.13) and using elementary estimates we get

$$
\begin{aligned}
\int_{0}^{t} \int_{\Gamma_{n}} g_{n}^{\prime}(\tau) u_{m}^{\prime \prime}(\tau) d S d \tau \leq & \int_{0}^{t}\left\|g_{n}^{\prime \prime}(\tau)\right\|_{H^{-1 / 2}\left(\Gamma_{n} ; \mathbb{R}^{3}\right)}\left\|u_{m}^{\prime}(\tau)\right\|_{H^{1}\left(\Omega ; \mathbb{R}^{3}\right)} d \tau \\
& +\left\|g_{n}^{\prime}(t)\right\|_{H^{-1 / 2}\left(\Gamma_{n} ; \mathbb{R}^{3}\right)}\left\|u_{m}^{\prime}(t)\right\|_{H^{1}\left(\Omega ; \mathbb{R}^{3}\right)} \\
& +\left\|g_{n}^{\prime}(0)\right\|_{H^{-1 / 2}\left(\Gamma_{n} ; \mathbb{R}^{3}\right)}\left\|u_{m}^{\prime}(0)\right\|_{H^{1}\left(\Omega ; \mathbb{R}^{3}\right)}
\end{aligned}
$$

The inequality (3.17) allows us to write

$$
\begin{aligned}
& \frac{\rho}{2}\left\|u_{m}^{\prime \prime}(t)\right\|_{L^{2}\left(\Omega ; \mathbb{R}^{3}\right)}^{2}+\frac{1}{2} \int_{\Omega} \mathcal{D}\left(\varepsilon\left(u_{m}^{\prime}(t)\right)\right) \varepsilon\left(u_{m}^{\prime}(t)\right) d x \\
& +c \int_{0}^{t}\left\|\nabla p_{m}^{\prime}(\tau)\right\|_{L^{2}\left(\Omega ; \mathbb{R}^{3}\right)}^{2} d \tau \leq \widetilde{C}(T, \nu)+\nu \sup _{t \in(0, T)}\left\|u_{m}^{\prime}(t)\right\|_{H^{1}\left(\Omega ; \mathbb{R}^{3}\right)}^{2} \\
& \quad+\nu \sup _{t \in(0, T)}\left\|u_{m}^{\prime \prime}(t)\right\|_{L^{2}\left(\Omega ; \mathbb{R}^{3}\right)}^{2}+\nu \int_{0}^{t}\left\|p_{m}^{\prime}(\tau)\right\|_{H^{1}(\Omega ; \mathbb{R})}^{2} d \tau
\end{aligned}
$$

where $\nu>0$ is any positive number and $\widetilde{C}(T, \nu)$ does not depend on $m$. We know that the function

$$
N(u)=\|\varepsilon(u)\|_{L^{2}\left(\Omega ; \mathcal{S}^{3}\right)}+\int_{\Gamma_{d}}|u| d S(x)
$$

is a norm on $H^{1}\left(\Omega ; \mathbb{R}^{3}\right)$ equivalent to the standard norm (see [20]). Using this fact, assumption about $\mathcal{D}$ and $u_{\left.m\right|_{\Gamma_{d}}}=p_{\left.m\right|_{\Gamma_{p}}}=0$ we can write that the left-hand side of (3.18) is equivalent to the norm

$$
\left\|u_{m}^{\prime \prime}(t)\right\|_{L^{2}\left(\Omega ; \mathbb{R}^{3}\right)}^{2}+\left\|u_{m}^{\prime}(t)\right\|_{H^{1}\left(\Omega ; \mathbb{R}^{3}\right)}^{2}+c \int_{0}^{t}\left\|p_{m}^{\prime}(\tau)\right\|_{H^{1}(\Omega ; \mathbb{R})}^{2} d \tau .
$$

Choosing $\nu>0$ sufficiently small we arrive at the inequality (3.12).

Step 4. Passing to the limit with $m \rightarrow \infty$.

The energy estimates proved in the last step yield that the sequences $u_{m}$ and $p_{m}$ are bounded in $W^{1, \infty}(0, T ; V)$ and $H^{1}(0, T ; W)$, respectively and the 
sequence $u_{m}^{\prime \prime}$ is bounded in $L^{\infty}\left(0, T ; L^{2}\left(\Omega ; \mathbb{R}^{3}\right)\right)$. Hence, for a subsequence (again denoted using the subscript $m$ ) we have

$$
\begin{array}{ll}
u_{m} \stackrel{*}{\rightarrow} u & \text { in } W^{1, \infty}(0, T ; V), \\
u_{m}^{\prime \prime} \stackrel{*}{\rightarrow} u^{\prime \prime} & \text { in } L^{\infty}\left(0, T ; L^{2}\left(\Omega ; \mathbb{R}^{3}\right)\right), \\
p_{m} \rightarrow p & \text { in } H^{1}(0, T ; W),
\end{array}
$$

for all $T>0$. Passing to the limit with $m \rightarrow \infty$ in (3.9) and (3.10) we obtain a solution of the system (3.1) in the sense of Definition 3.2. This part of the proof is a standard one in the Galerkin method and the details are left to the reader.

Step 5. Uniqueness.

THEOREM 3.6. Let us assume that the given data satisfy all requirements of Theorem 3.5. Then the weak solution of the system (3.1) is unique.

Proof. Assume that $\left(u^{1}, p^{1}\right)$ and $\left(u^{2}, p^{2}\right)$ are two weak solutions of (3.1) for the same given data. Let $u=u^{1}-u^{2}$ and $p=p^{1}-p^{2}$. From the regularity of the weak solution of the problem (3.1), we can take as a test function $v=u_{t}$ and $w=p$. Doing similar calculations to the ones from the beginning of the proof of Theorem 3.5 we easily get the inequality

$$
\left\|u^{\prime}(t)\right\|_{L^{2}\left(\Omega ; \mathbb{R}^{3}\right)}^{2}+\|u(t)\|_{H^{1}\left(\Omega ; \mathbb{R}^{3}\right)}^{2}+c \int_{0}^{t}\|p(\tau)\|_{H^{1}(\Omega ; \mathbb{R})}^{2} d \tau \leq 0 .
$$

This inequality finishes the proof of Theorems 3.6 and 3.3.

\section{Existence result for each approximation step}

In this section we approximate the problem (1.1) by a coercive and monotone problem. The monotone approximation to the non-monotone quasi-static model in poroplasticity was proposed in the article [18]. We use this monotone approximation to the non-monotone dynamic system in poroplasticity. To the system (1.1) we also use the coercive approximation, which can be found in [6]. Let $\eta>0$ and $\beta>1$, then the approximation is defined by

$$
\begin{aligned}
\rho u_{t t}^{\eta}(x, t)-\operatorname{div}_{x} T^{\eta}(x, t)+\nabla_{x} p^{\eta}(x, t) & =F(x, t), \\
c \Delta_{x} p^{\eta}(x, t)-\operatorname{div}_{x} u_{t}^{\eta}(x, t) & =f(x, t), \\
T^{\eta}(x, t) & =\mathcal{D}\left(\varepsilon^{\eta}(x, t)-\varepsilon^{p, \eta}(x, t)+\eta \varepsilon^{\eta}(x, t)\right), \\
\varepsilon^{\eta}(x, t) & =\frac{1}{2}\left(\nabla_{x} u^{\eta}(x, t)+\nabla_{x}^{T} u^{\eta}(x, t)\right), \\
\varepsilon_{t}^{p, \eta}(x, t) & =\eta\left|\widehat{T}^{\eta}(x, t)\right|^{\beta} \frac{\widehat{T}^{\eta}(x, t)}{\left|\widehat{T}^{\eta}(x, t)\right|} \\
& +\mathcal{F}\left(Y\left(\widehat{T}^{\eta}(x, t)\right)\right) \frac{\partial P}{\partial T}\left(\widehat{T}^{\eta}(x, t)\right) .
\end{aligned}
$$


Here, $\widehat{T}^{\eta}=\mathcal{D}\left(\varepsilon^{\eta}-\varepsilon^{p, \eta}\right)=T^{\eta}-\eta \mathcal{D} \varepsilon^{\eta}$. The approximate free energy function is given by the formula

$$
\rho \psi^{\eta}\left(\varepsilon^{\eta}, \varepsilon^{p, \eta}\right)=\frac{1}{2} \mathcal{D}\left(\varepsilon^{\eta}-\varepsilon^{p, \eta}\right)\left(\varepsilon^{\eta}-\varepsilon^{p, \eta}\right)+\frac{1}{2} \eta \mathcal{D} \varepsilon^{\eta} \varepsilon^{\eta} .
$$

The free energy function $\psi$ in (4.2) is now a quadratic positive-definite form. The system (4.1) is coercive for all $\eta>0$. The total energy of the system (4.1) is in the form

$$
\mathcal{E}^{\eta}\left(u_{t}^{\eta}, \varepsilon^{\eta}, \varepsilon^{p, \eta}\right)(t)=\frac{1}{2} \int_{\Omega} \rho\left|u_{t}^{\eta}(x, t)\right|^{2} d x+\int_{\Omega} \rho \psi^{\eta}\left(\varepsilon^{\eta}(x, t), \varepsilon^{p, \eta}(x, t)\right) d x .
$$

The problem (4.1) is considered with mixed boundary conditions:

$$
\begin{aligned}
u^{\eta}(x, t) & =g_{d}(x, t) & & \text { for } x \in \Gamma_{d} \text { and } t \geq 0 \\
\left(T^{\eta}(x, t)-p^{\eta}(x, t) I\right) n(x, t) & =g_{n}(x, t) & & \text { for } x \in \Gamma_{n} \text { and } t \geq 0 \\
p^{\eta}(x, t) & =g_{p}(x, t) & & \text { for } x \in \Gamma_{p} \text { and } t \geq 0 \\
c \frac{\partial p^{\eta}}{\partial n}(x, t) & =g_{v}(x, t) & & \text { for } x \in \Gamma_{v} \text { and } t \geq 0
\end{aligned}
$$

and with initial conditions:

$$
u^{\eta}(x, 0)=u^{0}(x), \quad u_{t}^{\eta}(x, 0)=u^{1}(x), \quad \varepsilon^{p, \eta}(x, 0)=\varepsilon^{p, 0}(x) .
$$

From Lemma 2.2 in [18] we know that the approximate nonlinear constitutive function in (4.1) has almost monotone structure which means: for $\beta>\max \left(1, r^{\prime}\right)$, where $\left.r^{\prime}=(\alpha-1) \operatorname{deg}(Y)(\operatorname{deg}(Y)-1)^{2}\right)$ it holds

$$
G^{\eta}(T)=\eta|T|^{\beta} \frac{T}{|T|}+\mathcal{F}(Y(T)) \frac{\partial P}{\partial T}(T) \in \mathcal{L} \mathcal{M}
$$

for all $\eta>0$ and $T \in \mathcal{S}^{3}$, where the class $\mathcal{L} \mathcal{M}$ is the class of Lipschitz perturbations of maximal monotone vector fields (for definition see [8] or [18]). In the next part of this section we are going to prove the existence and uniqueness of the solution of the system (4.1) with initial-boundary conditions (4.2)-(4.3) for each $\eta>0$. The nonlinear function in (4.1) belongs to the $\mathcal{L} \mathcal{M}$ class. Thus $G^{\eta}$ can be written in the form $G^{\eta}=g^{\eta}+\mathcal{L}^{\eta}$, where $g^{\eta}: \mathcal{S}^{3} \rightarrow \mathcal{S}^{3}$ is a monotone field and $\mathcal{L}^{\eta}: \mathcal{S}^{3} \rightarrow \mathcal{S}^{3}$ is a global Lipschitz operator. To prove the existence for the system (4.1) we use Yosida approximation for $g^{\eta}$. We rewrite system (4.1) with $G^{\eta, \lambda}=g^{\eta, \lambda}+\mathcal{L}^{\eta}$ instead of $G^{\eta}$, where $g^{\eta, \lambda}=\lambda^{-1}\left(I-J_{\lambda}\right)$ and $J_{\lambda}=\left(I+\lambda g^{\eta}\right)^{-1}$. Next, we pass to the limit $\lambda \rightarrow 0^{+}$. For $\lambda>0$ we consider the following system 
of equations

$$
\begin{aligned}
& \rho u_{t t}^{\eta, \lambda}(x, t)-\operatorname{div}_{x} T^{\eta, \lambda}(x, t)+\nabla_{x} p^{\eta, \lambda}(x, t)=F(x, t), \\
& c \Delta_{x} p^{\eta, \lambda}(x, t)-\operatorname{div}_{x} u_{t}^{\eta, \lambda}(x, t)=f(x, t), \\
& T^{\eta, \lambda}(x, t)=\mathcal{D}\left(\varepsilon^{\eta, \lambda}(x, t)-\varepsilon^{p, \eta, \lambda}(x, t)\right. \\
&\left.+\eta \varepsilon^{\eta, \lambda}(x, t)\right), \\
&5) \quad \frac{1}{2}\left(\nabla_{x} u^{\eta, \lambda}(x, t)+\nabla_{x}^{T} u^{\eta, \lambda}(x, t)\right), \\
& \varepsilon^{\eta, \lambda}(x, t) \varepsilon_{t}^{p, \eta, \lambda}(x, t)= \\
& G^{\eta, \lambda}\left(\widehat{T}^{\eta, \lambda}(x, t)\right),
\end{aligned}
$$

with the same given forces, boundary conditions and initial conditions as for the system (4.1).

In the next part of this section, we will drop the superscript $\eta>0$ and write $u^{\lambda}, \varepsilon^{p, \lambda}, p^{\lambda}$ instead of $u^{\eta, \lambda}, \varepsilon^{p, \eta, \lambda}, p^{\eta, \lambda}$.

THEOREM 4.1. Assume that the given data possesses the regularity required in (2.1)-(2.5) and that the compatibility condition holds. Then, for all $\lambda>0$ the approximate problem (4.5) has a global in time, unique solution $\left(u^{\lambda}, p^{\lambda}, \varepsilon^{p, \lambda}\right)$ with the regularity

$$
\begin{array}{ll}
u^{\lambda} \in W^{1, \infty}\left(0, T ; H^{1}\left(\Omega ; \mathbb{R}^{3}\right)\right), & u_{t t}^{\lambda} \in L^{\infty}\left(0, T ; L^{2}\left(\Omega ; \mathbb{R}^{3}\right)\right), \\
p^{\lambda} \in H^{1}\left(0, T ; H^{1}(\Omega ; \mathbb{R})\right), & \varepsilon^{p, \lambda} \in W^{1, \infty}\left(0, T ; L^{2}\left(\Omega ; \mathcal{S}^{3}\right)\right) .
\end{array}
$$

Proof. The system (4.5) contains only global Lipschitz nonlinearities and the proof immediately follows from the Banach Fixed Point Theorem and we give a sketch of the proof. For a fixed $T>0$ we construct an operator

$$
P: C\left([0, T] ; L^{2}\left(\Omega ; \mathcal{S}^{3}\right)\right) \rightarrow C\left([0, T] ; L^{2}\left(\Omega ; \mathcal{S}^{3}\right)\right)
$$

as follows: for $\varepsilon \in C\left([0, T] ; L^{2}\left(\Omega ; \mathcal{S}^{3}\right)\right)$ let us consider the equation

$$
\varepsilon^{p}(t)=\varepsilon^{p, 0}+\int_{0}^{t} G^{\eta, \lambda}\left(\mathcal{D}\left(\varepsilon(\tau)-\varepsilon^{p}(\tau)\right)\right) d \tau .
$$

By the regularity of $G^{\eta, \lambda}$ it follows that this equation possesses global in time, unique solution $\varepsilon^{p} \in C^{1}\left([0, T] ; L^{2}\left(\Omega ; \mathcal{S}^{3}\right)\right)$. For the solution $\varepsilon^{p}$ we consider the following system of equations

$$
\begin{array}{r}
\rho u_{t t}-\operatorname{div}_{x}\left(\mathcal{D}\left(\varepsilon-\varepsilon^{p}\right)\right)+\nabla_{x} p=F, \\
c \Delta_{x} p-\operatorname{div}_{x} u_{t}=f,
\end{array}
$$

with boundary conditions and initial conditions as for the system (4.5). The system above is the classical dynamic Biot model, which was considered in the 
last section. This problem has a unique solution $u \in C\left([0, T] ; H^{1}\left(\Omega ; \mathbb{R}^{3}\right)\right)$ and $p \in C\left([0, T] ; H^{1}(\Omega ; \mathbb{R})\right)$. Finally, we set

$$
P(\varepsilon)=\frac{1}{2}\left(\nabla u+\nabla^{T} u\right) .
$$

Let us denote by $\varepsilon_{1}^{p}(t)$ and $\varepsilon_{2}^{p}(t)$ solutions of (4.6) with the input functions $\varepsilon_{1}, \varepsilon_{2} \in C\left([0, T] ; L^{2}\left(\Omega ; \mathcal{S}^{3}\right)\right)$, respectively. By direct inspection of (4.7) we have

$$
\left\|P\left(\varepsilon_{1}\right)(t)-P\left(\varepsilon_{2}\right)(t)\right\|_{L^{2}\left(\Omega ; \mathcal{S}^{3}\right)} \leq C\left\|\varepsilon_{1}^{p}(t)-\varepsilon_{2}^{p}(t)\right\|_{L^{2}\left(\Omega ; \mathcal{S}^{3}\right)},
$$

where the positive constant $C$ does not depend on these input functions and is independent of $t$. Using the equation (4.6) it is not difficult to obtain the following inequality

$$
\left\|\varepsilon_{1}^{p}(t)-\varepsilon_{2}^{p}(t)\right\|_{L^{2}\left(\Omega ; \mathcal{S}^{3}\right)} \leq \widetilde{C}\left\|\varepsilon_{1}(t)-\varepsilon_{2}(t)\right\|_{L^{2}\left(\Omega ; \mathcal{S}^{3}\right)},
$$

where $\widetilde{C}$ does not depend on $t$ (it depends only on the Lipschitz constant and on time $T$ ). Having this two inequalities we easily see that the operator $P$ is a contraction (for similar results we refer to [17]). From the definition of operator $P$ and Theorem 3.3 we conclude that the solution $\left(u^{\lambda}, p^{\lambda}, \varepsilon^{p, \lambda}\right)$ has the regularity required in the statement of theorem.

Remark 4.2. Notice that from Theorems 3.3 and 4.1 we get that $u_{t}^{\lambda} \in$ $L^{\infty}\left(0, T ; H^{1}\left(\Omega ; \mathbb{R}^{3}\right)\right)$. Having this information we will obtain that

$$
u_{t}^{\eta} \in L^{\infty}\left(0, T ; H^{1}\left(\Omega ; \mathbb{R}^{3}\right)\right) \text { for all } \eta>0 .
$$

This regularity is better than the one obtained in [18]. In article [18] for the quasi-static case we only obtain that $u_{t}^{\eta} \in L^{2}\left(0, T ; H^{1}\left(\Omega ; \mathbb{R}^{3}\right)\right)$ and to prove it we had to apply the method, which uses the structure of the approximate constitutive function (for details, see [16] and [17]). In the next part of this section we prove some estimates for the sequence $\left(u^{\lambda}, p^{\lambda}, \varepsilon^{p, \lambda}\right)$. To do this we only use the energy method and the fact that the function $G^{\eta} \in \mathcal{L} \mathcal{M}$.

From the assumption on the given boundary data $g_{p}$, there exists a function $p^{*} \in W^{2, \infty}\left(0, T ; H^{2}(\Omega ; \mathbb{R})\right)$ such that $\left.p^{*}\right|_{\Gamma_{p}}=g_{\left.p\right|_{\Gamma_{p}}}$ and the system (4.5) can be written in the form:

$$
\begin{aligned}
\rho u_{t t}^{\lambda}(x, t)-\operatorname{div}_{x} T^{\lambda}(x, t)+\nabla_{x}\left(p^{\lambda}(x, t)-p^{*}(x, t)\right) & =F(x, t)-\nabla_{x} p^{*}(x, t), \\
c \Delta_{x}\left(p^{\lambda}(x, t)-p^{*}(x, t)\right)-\operatorname{div}_{x} u_{t}^{\lambda}(x, t) & =f(x, t)-c \Delta_{x} p^{*}(x, t), \\
\varepsilon_{t}^{p, \lambda}(x, t) & =G^{\lambda}\left(\widehat{T}^{\lambda}(x, t)\right) .
\end{aligned}
$$


ThEOREM 4.3 (Energy estimate for time derivatives). Suppose that our data have the regularity required in $(2.1)-(2.5)$. Assume that $g^{\eta}\left(\mathcal{D}\left(\varepsilon\left(u^{0}\right)-\varepsilon^{p, 0}\right)\right) \in$ $L^{2}\left(\Omega ; \mathcal{S}^{3}\right)$ for all $\eta>0$. Then, for all $T>0$ there exists a positive constant $C(T)$ independent of $\lambda$ such that the inequality

$$
\mathcal{E}^{\eta}\left(v_{t}^{\lambda}, \varepsilon_{t}^{\lambda}, \varepsilon_{t}^{p, \lambda}\right)(t)+c \int_{0}^{t} \int_{\Omega}\left|\nabla\left(p_{t}^{\lambda}-p_{t}^{*}\right)\right|^{2} d x d \tau \leq C(T)
$$

holds for all $t \in[0, T)$.

Proof. For $h>0$ let us denote by $\left(v_{h}^{\lambda}, \varepsilon_{h}^{\lambda}, \varepsilon_{h}^{p, \lambda}\right)$ the shifted functions $\left(v^{\lambda}(t+\right.$ $\left.h), \varepsilon^{\lambda}(t+h), \varepsilon_{h}^{p, \lambda}(t+h)\right)$ where $v^{\lambda}=u_{t}^{\lambda}$. Calculating the time derivative of the energy evaluated on the differences $\left(v_{h}^{\lambda}-v^{\lambda}, \varepsilon_{h}^{\lambda}-\varepsilon^{\lambda}, \varepsilon_{h}^{p, \lambda}-\varepsilon^{p, \lambda}\right)$, we arrive at the inequality

$$
\begin{aligned}
& \frac{d}{d t} \mathcal{E}^{\eta}\left(v_{h}^{\lambda}-v^{\lambda}, \varepsilon_{h}^{\lambda}-\varepsilon^{\lambda}, \varepsilon_{h}^{p, \lambda}-\varepsilon^{p, \lambda}\right)=\rho \int_{\Omega}\left(v_{h}^{\lambda}-v^{\lambda}\right)\left(v_{h, t}^{\lambda}-v_{t}^{\lambda}\right) d x \\
& \quad+\int_{\Omega}\left(T_{h}^{\lambda}-T^{\lambda}\right)\left(\varepsilon_{h, t}^{\lambda}-\varepsilon_{t}^{\lambda}\right) d x-\int_{\Omega}\left(\widehat{T}_{h}^{\lambda}-\widehat{T}^{\lambda}\right)\left(\varepsilon_{h, t}^{p, \lambda}-\varepsilon_{t}^{p, \lambda}\right) d x \\
& =\rho \int_{\Omega}\left(v_{h}^{\lambda}-v^{\lambda}\right)\left(v_{h, t}^{\lambda}-v_{t}^{\lambda}\right) d x+\int_{\Omega}\left(T_{h}^{\lambda}-T^{\lambda}\right)\left(\nabla v_{h}^{\lambda}-\nabla v^{\lambda}\right) d x \\
& \quad-\left(\int_{\Omega}\left(\widehat{T}_{h}^{\lambda}-\widehat{T}^{\lambda}\right)\left(\varepsilon_{h, t}^{p, \lambda}-\varepsilon_{t}^{p, \lambda}\right) d x+L \int_{\Omega}\left|\widehat{T}_{h}^{\lambda}-\widehat{T}^{\lambda}\right|^{2} d x\right) \\
& \quad+L \int_{\Omega}\left|\widehat{T}_{h}^{\lambda}-\widehat{T}^{\lambda}\right|^{2} d x
\end{aligned}
$$

(by integrating by parts in the second term and $G^{\eta} \in \mathcal{L} \mathcal{M}$ )

$$
\begin{aligned}
\leq & \int_{\Omega}\left(F_{h}-F-\nabla\left(p_{h}^{*}-p^{*}\right)\right)\left(v_{h}^{\lambda}-v^{\lambda}\right) d x \\
& +\int_{\partial \Omega}\left(T_{h}^{\lambda}-T^{\lambda}\right) n\left(v_{h}^{\lambda}-v^{\lambda}\right) d S(x) \\
& -\int_{\Omega} \nabla\left(p_{h}^{\lambda}-p^{\lambda}-\left(p_{h}^{*}-p^{*}\right)\right)\left(v_{h}^{\lambda}-v^{\lambda}\right) d x+L \int_{\Omega}\left|\widehat{T}_{h}^{\lambda}-\widehat{T}^{\lambda}\right|^{2} d x,
\end{aligned}
$$

where $T_{h}^{\lambda}=T^{\lambda}(t+h), p_{h}^{\lambda}=p^{\lambda}(t+h), p_{h}^{*}=p^{*}(t+h)$ and $F_{h}=F(t+h)$. Integrating twice by parts in the third integral on the right hand-side of (4.9) we obtain

$$
\begin{aligned}
& \frac{d}{d t} \mathcal{E}^{\eta}\left(v_{h}^{\lambda}-v^{\lambda}, \varepsilon_{h}^{\lambda}-\varepsilon^{\lambda}, \varepsilon_{h}^{p, \lambda}-\varepsilon^{p, \lambda}\right)+c \int_{\Omega}\left|\nabla\left(p_{h}^{\lambda}-p^{\lambda}-\left(p_{h}^{*}-p^{*}\right)\right)\right|^{2} d x \\
& \leq \int_{\Omega}\left(F_{h}-F-\nabla\left(p_{h}^{*}-p^{*}\right)\right)\left(v_{h}^{\lambda}-v^{\lambda}\right) d x \\
& \quad+\int_{\partial \Omega}\left(T_{h}^{\lambda}-I p_{h}^{\lambda}-\left(T^{\lambda}-I p^{\lambda}\right)\right) n\left(v_{h}^{\lambda}-v^{\lambda}\right) d S(x) \\
& \quad+\int_{\partial \Omega}\left(p_{h}^{*}-p^{*}\right) \operatorname{In}\left(v_{h}^{\lambda}-v^{\lambda}\right) d S(x)
\end{aligned}
$$




$$
\begin{aligned}
& -\int_{\Omega}\left(f_{h}-f-c\left(\Delta\left(p_{h}^{*}-p^{*}\right)\right)\right)\left(p_{h}^{\lambda}-p^{\lambda}-\left(p_{h}^{*}-p^{*}\right)\right) d x \\
& +c \int_{\partial \Omega}\left(p_{h}^{\lambda}-p^{\lambda}-\left(p_{h}^{*}-p^{*}\right)\right) \frac{\partial\left(p_{h}^{\lambda}-p^{\lambda}\right)}{\partial n} d S(x) \\
& -c \int_{\partial \Omega}\left(p_{h}^{\lambda}-p^{\lambda}-\left(p_{h}^{*}-p^{*}\right)\right) \frac{\partial\left(p_{h}^{*}-p^{*}\right)}{\partial n} d S(x)+L \int_{\Omega}\left|\widehat{T}_{h}^{\lambda}-\widehat{T}^{\lambda}\right|^{2} d x .
\end{aligned}
$$

Dividing by $h^{2}$, using boundary data and integrating the whole inequality (4.10) with respect to time we have

$$
\begin{aligned}
& \frac{1}{h^{2}} \mathcal{E}^{\eta}\left(v_{h}^{\lambda}-v^{\lambda}, \varepsilon_{h}^{\lambda}-\varepsilon^{\lambda}, \varepsilon_{h}^{p, \lambda}-\varepsilon^{p, \lambda}\right)(t) \\
& \quad+c \frac{1}{h^{2}} \int_{0}^{t} \int_{\Omega}\left|\nabla\left(p_{h}^{\lambda}-p^{\lambda}-\left(p_{h}^{*}-p^{*}\right)\right)\right|^{2} d x \\
& \leq \frac{1}{h^{2}} \mathcal{E}^{\eta}\left(v_{h}^{\lambda}-v^{\lambda}, \varepsilon_{h}^{\lambda}-\varepsilon^{\lambda}, \varepsilon_{h}^{p, \lambda}-\varepsilon^{p, \lambda}\right)(0) \\
&+\frac{1}{h^{2}} \int_{0}^{t} \int_{\Omega}\left(F_{h}-F-\nabla\left(p_{h}^{*}-p^{*}\right)\right)\left(v_{h}^{\lambda}-v^{\lambda}\right) d x d \tau \\
&+\frac{1}{h^{2}} \int_{0}^{t} \int_{\Gamma_{d}}\left(T_{h}^{\lambda}-T^{\lambda}-I\left(p_{h}^{\lambda}-p^{\lambda}\right)\right) n\left(\partial_{t} g_{d, h}-\partial_{t} g_{d}\right) d S(x) d \tau \\
&+\frac{1}{h^{2}} \int_{0}^{t} \int_{\Gamma_{n}}\left(g_{n, h}-g_{n}\right)\left(v_{h}^{\lambda}-v^{\lambda}\right) d S(x) d \tau \\
&+\frac{1}{h^{2}} \int_{0}^{t} \int_{\partial \Omega}\left(p_{h}^{*}-p^{*}\right) \operatorname{In}\left(v_{h}^{\lambda}-v^{\lambda}\right) d S(x) d \tau \\
&-\frac{1}{h^{2}} \int_{0}^{t} \int_{\Omega}\left(f_{h}-f-c\left(\Delta\left(p_{h}^{*}-p^{*}\right)\right)\right)\left(p_{h}^{\lambda}-p^{\lambda}-\left(p_{h}^{*}-p^{*}\right)\right) d x d \tau \\
&+\frac{1}{h^{2}} \int_{0}^{t} \int_{\Gamma_{v}}\left(p_{h}^{\lambda}-p^{\lambda}-\left(p_{h}^{*}-p^{*}\right)\right)\left(g_{v, h}-g_{v}\right) d S(x) d \tau \\
&-c \frac{1}{h^{2}} \int_{0}^{t} \int_{\partial \Omega}\left(p_{h}^{\lambda}-p^{\lambda}-\left(p_{h}^{*}-p^{*}\right)\right) \frac{\partial\left(p_{h}^{*}-p^{*}\right)}{\partial n} d S(x) d \tau \\
&+\left.L \frac{1}{h^{2}} \int_{0}^{t} \int_{\Omega}\left|\widehat{T}_{h}^{\lambda}-\widehat{T}^{\lambda}\right|\right|^{2} d x d \tau,
\end{aligned}
$$

where $g_{d, h}=g_{d}(t+h), g_{n, h}=g_{n}(t+h), f_{h}=f(t+h)$ and $g_{v, h}=g_{v}(t+h)$.

Now we shift the difference operator from the functions $v^{\lambda}, T^{\lambda}$ onto the given data with details for the fourth integral on the right hand-side of (4.11) only.

$$
\begin{aligned}
& \frac{1}{h^{2}} \int_{0}^{t} \int_{\Gamma_{n}}\left(g_{n, h}-g_{n}\right)\left(v_{h}^{\lambda}-v^{\lambda}\right) d S(x) d \tau \\
& =\frac{1}{h^{2}} \int_{0}^{t} \int_{\Gamma_{n}}\left(g_{n, h}-g_{n}\right) v_{h}^{\lambda} d S(x) d \tau-\frac{1}{h^{2}} \int_{0}^{t} \int_{\Gamma_{n}}\left(g_{n, h}-g_{n}\right) v^{\lambda} d S(x) d \tau
\end{aligned}
$$


$(\tau+h=s$ in the first integral $)$

$$
\begin{aligned}
= & \frac{1}{h^{2}} \int_{h}^{t+h} \int_{\Gamma_{n}}\left(g_{n}(s)-g_{n}(s-h)\right) v^{\lambda} d S(x) d s \\
& -\frac{1}{h^{2}} \int_{0}^{t} \int_{\Gamma_{n}}\left(g_{n, h}-g_{n}\right) v^{\lambda} d S(x) d \tau \\
= & -\frac{1}{h^{2}} \int_{h}^{t} \int_{\Gamma_{n}}\left(g_{n}(s+h)-2 g_{n}(s)+g_{n}(s-h)\right) v^{\lambda} d S(x) d \tau \\
& -\frac{1}{h^{2}} \int_{0}^{h} \int_{\Gamma_{n}}\left(g_{n, h}-g_{n}\right) v^{\lambda} d S(x) d \tau \\
& +\frac{1}{h^{2}} \int_{t}^{t+h} \int_{\Gamma_{n}}\left(g_{n}(s)-g_{n}(s-h)\right) v^{\lambda} d S(x) d s .
\end{aligned}
$$

The last two integrals from (4.12) we can estimate by the following expression

$$
\int_{0}^{T}\left\|\frac{1}{h}\left(g_{n, h}-g_{n}\right)\right\|_{H^{-1 / 2}\left(\partial \Omega ; \mathbb{R}^{3}\right)}\left\|v^{\lambda}(\tau)\right\|_{H^{1 / 2}\left(\partial \Omega ; \mathbb{R}^{3}\right)} \cdot \frac{1}{h} \chi_{F}(\tau) d \tau
$$

where $\chi_{F}$ denotes the characteristic function of some $F$ such that $\operatorname{diam}(F)=h$. Doing similar calculations as in (4.12) with the third and fifth terms on the right hand-side of (4.11), using regularity on the given data and $v^{\lambda}$ we can pass to the limit with $h \rightarrow 0^{+}$in (4.11) and we get the inequality

$$
\begin{aligned}
& \mathcal{E}^{\eta}\left(v_{t}^{\lambda}, \varepsilon_{t}^{\lambda}, \varepsilon_{t}^{p, \lambda}\right)(t)+c \int_{0}^{t} \int_{\Omega}\left|\nabla\left(p_{t}^{\lambda}-p_{t}^{*}\right)\right|^{2} d x \leq \mathcal{E}^{\eta}\left(v_{t}^{\lambda}, \varepsilon_{t}^{\lambda}, \varepsilon_{t}^{p, \lambda}\right)(0) \\
& \quad+\int_{0}^{t} \int_{\Omega}\left(\left\|F_{t}\right\|_{L^{2}\left(\Omega ; \mathbb{R}^{3}\right)}+\left\|\nabla p_{t}^{*}\right\|_{L^{2}\left(\Omega ; \mathbb{R}^{3}\right)}\right)\left\|v_{t}^{\lambda}\right\|_{L^{2}\left(\Omega ; \mathbb{R}^{3}\right)} d \tau \\
& \quad+C(T)\left(\sup _{t \in(0, T)}\left\|\partial_{t t t} g_{d}(t)\right\|_{H^{1 / 2}\left(\partial \Omega ; \mathbb{R}^{3}\right)}\right. \\
& \left.\quad+\sup _{t \in(0, T)}\left\|\partial_{t t} g_{d}(t)\right\|_{H^{1 / 2}\left(\partial \Omega ; \mathbb{R}^{3}\right)}\right) \sup _{t \in(0, T)}\left\|\left(T^{\lambda}-I p^{\lambda}\right) n\right\|_{H^{-1 / 2}\left(\partial \Omega ; \mathbb{R}^{3}\right)} \\
& \quad+C(T)\left(\sup _{t \in(0, T)}\left\|\partial_{t t} g_{n}(t)\right\|_{H^{-1 / 2}\left(\partial \Omega ; \mathbb{R}^{3}\right)}+\sup _{t \in(0, T)}\left\|\partial_{t} g_{n}(t)\right\|_{H^{-1 / 2}\left(\partial \Omega ; \mathbb{R}^{3}\right)}\right. \\
& \left.\quad+\sup _{t \in(0, T)}\left\|\partial_{t t} p^{*}(t)\right\|_{H^{-1 / 2}(\partial \Omega ; \mathbb{R})}+\sup _{t \in(0, T)}\left\|\partial_{t} p^{*}(t)\right\|_{H^{-1 / 2}(\partial \Omega ; \mathbb{R})}\right) \\
& \quad+\sup _{t \in(0, T)}\left\|v^{\lambda}(t)\right\|_{H^{1 / 2}\left(\partial \Omega ; \mathbb{R}^{3}\right)} \\
& \quad+\int_{0}^{t}\left(\left\|f_{t}\right\|_{L^{2}(\Omega ; \mathbb{R})}+c\left\|p_{t}^{*}\right\|_{H^{2}(\Omega ; \mathbb{R})}\right)\left\|p_{t}^{\lambda}-p_{t}^{*}\right\|_{L^{2}(\Omega ; \mathbb{R})} d \tau \\
& \quad+\int_{0}^{t}\left\|p_{t}^{\lambda}-p_{t}^{*}\right\|_{H^{1 / 2}(\partial \Omega ; \mathbb{R})}\left\|\partial_{t} g_{v}\right\|_{H^{-1 / 2}(\partial \Omega ; \mathbb{R})} d \tau
\end{aligned}
$$




$$
\begin{aligned}
& +c \int_{0}^{t}\left\|p_{t}^{\lambda}-p_{t}^{*}\right\|_{H^{1 / 2}(\partial \Omega ; \mathbb{R})}\left\|\nabla p_{t}^{*}\right\|_{H^{-1 / 2}(\partial \Omega ; \mathbb{R})} d \tau \\
& +L \int_{0}^{t}\left\|\widehat{T}_{t}^{\lambda}\right\|_{L^{2}\left(\Omega ; \mathcal{S}^{3}\right)}^{2} d \tau .
\end{aligned}
$$

The boundary norms appearing on the right-hand side of inequality (4.13) are estimated using the continuity of the trace operator

$$
\begin{aligned}
\|\left(T^{\lambda}-I p^{\lambda}\right) & n \|_{H^{-1 / 2}\left(\partial \Omega ; \mathbb{R}^{3}\right)} \\
\leq & C\left(\left\|T^{\lambda}-I p^{\lambda}\right\|_{L^{2}\left(\Omega ; \mathcal{S}^{3}\right)}+\left\|\operatorname{div}\left(T^{\lambda}-I p^{\lambda}\right)\right\|_{L^{2}\left(\Omega ; \mathbb{R}^{3}\right)}\right) \\
\leq & C\left(\left\|T^{\lambda}-I p^{\lambda}\right\|_{L^{2}\left(\Omega ; \mathcal{S}^{3}\right)}+\|F\|_{L^{2}\left(\Omega ; \mathbb{R}^{3}\right)}+\rho\left\|v_{t}^{\lambda}\right\|_{L^{2}\left(\Omega ; \mathbb{R}^{3}\right)}\right) \\
\leq & C\left(\left\|T^{\lambda}(0)-I p^{\lambda}(0)\right\|_{L^{2}\left(\Omega ; \mathcal{S}^{3}\right)}+T \sup _{t \in(0, T)}\left\|T_{t}^{\lambda}\right\|_{L^{2}\left(\Omega ; \mathcal{S}^{3}\right)}\right. \\
& \left.+\int_{0}^{t}\left\|p_{t}^{\lambda}\right\|_{H^{1}(\Omega ; \mathbb{R})} d \tau+\|F\|_{L^{2}\left(\Omega ; \mathbb{R}^{3}\right)}+\rho\left\|v_{t}^{\lambda}\right\|_{L^{2}\left(\Omega ; \mathbb{R}^{3}\right)}\right), \\
\left\|v^{\lambda}\right\|_{H^{\frac{1}{2}}\left(\partial \Omega ; \mathbb{R}^{3}\right)} \leq & C\left\|v^{\lambda}\right\|_{H^{1}\left(\Omega ; \mathbb{R}^{3}\right)} .
\end{aligned}
$$

From (4.12) and standard estimates we obtain

$$
\begin{aligned}
\mathcal{E}^{\eta}\left(v_{t}^{\lambda}, \varepsilon_{t}^{\lambda}, \varepsilon_{t}^{p, \lambda}\right)(t)+c \int_{0}^{t} \int_{\Omega}\left|\nabla\left(p_{t}^{\lambda}-p_{t}^{*}\right)\right|^{2} d x \leq \mathcal{E}^{\eta}\left(v_{t}^{\lambda}, \varepsilon_{t}^{\lambda}, \varepsilon_{t}^{p, \lambda}\right)(0) \\
+C(T, \nu)+\nu \sup _{t \in(0, T)}\left\|v_{t}^{\lambda}\right\|_{L^{2}\left(\Omega ; \mathbb{R}^{3}\right)}^{2}+\nu \sup _{t \in(0, T)}\left\|v^{\lambda}\right\|_{H^{1}\left(\Omega ; \mathbb{R}^{3}\right)}^{2} \\
+\left\|T^{\lambda}(0)-I p^{\lambda}(0)\right\|_{L^{2}\left(\Omega ; \mathcal{S}^{3}\right)}^{2}+\nu \sup _{t \in(0, T)}\left\|\widehat{T}_{t}^{\lambda}\right\|_{L^{2}\left(\Omega ; \mathcal{S}^{3}\right)} \\
+\nu \int_{0}^{t}\left\|p_{t}^{\lambda}-p_{t}^{*}\right\|_{H^{1}(\Omega ; \mathbb{R})}^{2} d \tau+L \int_{0}^{t}\left\|\widehat{T}_{t}^{\lambda}\right\|_{L^{2}\left(\Omega ; \mathcal{S}^{3}\right)}^{2} d \tau
\end{aligned}
$$

where $\nu>0$ is any positive number and $C(T, \nu)$ does not depend on $\lambda$.

Note that from the regularity of the initial conditions we obtain that the elliptic equation

$$
c \Delta_{x} p^{\lambda}(x, 0)-\operatorname{div}_{x} u^{1}(x)=f(x, 0)
$$

with boundary conditions

$$
\begin{array}{rlrl}
p^{\lambda}(x, 0) & =g_{p}(x, 0) & & \text { for } x \in \Gamma_{p}, \\
c \frac{\partial p^{\lambda}}{\partial n}(x, 0) & =g_{v}(x, 0) & \text { for } x \in \Gamma_{v},
\end{array}
$$

has a solution $p^{\lambda}(0) \in H^{1}(\Omega ; \mathbb{R})$ and this solution satisfies the following inequality

$$
\begin{aligned}
\left\|p^{\lambda}(0)\right\|_{H^{1}(\Omega ; \mathbb{R})} \leq C(\Omega)\left(\|f(0)\|_{L^{2}(\Omega ; \mathbb{R})}+\left\|\operatorname{div}_{x} u^{1}\right\|_{L^{2}(\Omega ; \mathbb{R})}\right. \\
\left.+\left\|g_{p}(0)\right\|_{H^{1 / 2}\left(\Gamma_{p} ; \mathbb{R}\right)}+\left\|g_{v}(0)\right\|_{H^{-1 / 2}\left(\Gamma_{v} ; \mathbb{R}\right)}\right) .
\end{aligned}
$$

From the assumption $g^{\eta}\left(\mathcal{D}\left(\varepsilon\left(u^{0}\right)-\varepsilon^{p, 0}\right)\right) \in L^{2}\left(\Omega ; \mathcal{S}^{3}\right)$ we conclude that the initial value of the energy evaluated for time derivatives is bounded. Choosing $\nu>0$ 
sufficiently small we get

$$
\mathcal{E}^{\eta}\left(v_{t}^{\lambda}, \varepsilon_{t}^{\lambda}, \varepsilon_{t}^{p, \lambda}\right)(t)+c \int_{0}^{t} \int_{\Omega}\left|\nabla\left(p_{t}^{\lambda}-p_{t}^{*}\right)\right|^{2} d x \leq C(T)+L \int_{0}^{t}\left\|\widehat{T}_{t}^{\lambda}\right\|_{L^{2}(\Omega)}^{2} d \tau
$$

The Gronwall inequality completes the proof.

Theorem 4.3 yields that the sequence of stresses $\left\{\widehat{T}^{\lambda}\right\}$ is bounded in $W^{1, \infty}(0$, $\left.T ; L^{2}\left(\Omega ; \mathcal{S}^{3}\right)\right)$. This information is not enough to pass to the limit in the system (4.5) with $\lambda \rightarrow 0^{+}$, because the Lipschitz constant of nonlinearities grows to infinity. We improve the convergence of the sequence $\left\{\widehat{T}^{\lambda}\right\}$.

THEOREM 4.4 (Strong convergence of stresses). Let us assume that the given data satisfy all requirements of Theorem 4.3. Then,

$$
\mathcal{E}^{\eta}\left(u^{\lambda}-u^{\mu}, \varepsilon^{\lambda}-\varepsilon^{\mu}, \varepsilon^{p, \lambda}-\varepsilon^{p, \mu}\right)(t)+c \int_{0}^{t} \int_{\Omega}\left|\nabla\left(p^{\lambda}-p^{\mu}\right)\right|^{2} d x d \tau \rightarrow 0
$$

for $\lambda, \mu \rightarrow 0^{+}$uniformly on bounded time intervals.

Proof. Calculating the time derivative of the energy evaluated on the difference of two approximate solutions and using the fact that the given data for two approximation steps are equal, we conclude that

$$
\begin{aligned}
\frac{d}{d t}\left(\mathcal{E}^{\eta}\left(u^{\lambda}-u^{\mu}, \varepsilon^{\lambda}-\varepsilon^{\mu}, \varepsilon^{p, \lambda}-\varepsilon^{p, \mu}\right)(t)\right)+c \int_{\Omega}\left|\nabla\left(p^{\lambda}-p^{\mu}\right)\right|^{2} d x & \\
& =-\int_{\Omega}\left(G^{\lambda}\left(\widehat{T}^{\lambda}\right)-G^{\mu}\left(\widehat{T}^{\mu}\right)\right)\left(\widehat{T}^{\lambda}-\widehat{T}^{\mu}\right) d x .
\end{aligned}
$$

Next, using the standard method for maximal monotone operators (see [4]) we easily get the inequality (the details can be found in [3] or [17])

$$
\begin{array}{r}
\mathcal{E}^{\eta}\left(u^{\lambda}-u^{\mu}, \varepsilon\left(u^{\lambda}\right)-\varepsilon\left(u^{\mu}\right), \varepsilon^{p, \lambda}-\varepsilon^{p, \mu}\right)(t)+c \int_{0}^{t} \int_{\Omega}\left|\nabla\left(p^{\lambda}-p^{\mu}\right)\right|^{2} d x d \tau \\
\leq \frac{1}{2}(\lambda+\mu) C(T),
\end{array}
$$

where the positive constant $C(T)$ is from Theorem 4.3 and it does not depend on $\lambda$ and $\mu$. The last inequality finishes the proof.

Finally, we formulate the main theorem of this section.

THEOREM 4.5 (Existence for each monotone approximation step). Suppose that the given data satisfy the regularity required in (2.1)-(2.5). Moreover, assume that the initial data satisfy the compatibility conditions and

$$
g^{\eta}\left(\mathcal{D}\left(\varepsilon\left(u^{0}\right)-\varepsilon^{p, 0}\right)\right) \in L^{2}\left(\Omega ; \mathcal{S}^{3}\right) \quad \text { for all } \eta>0 .
$$


Then, for all $\eta>0$, the system (4.1) with initial-boundary conditions (4.3)-(4.4) possesses a global in time, unique solution $\left(u^{\eta}, p^{\eta}, \varepsilon^{p, \eta}\right)$ with the regularity: for all $T>0$

$$
\begin{aligned}
u^{\eta} & \in W^{1, \infty}\left(0, T ; H^{1}\left(\Omega ; \mathbb{R}^{3}\right)\right), & u_{t t}^{\eta} & \in L^{\infty}\left(0, T ; L^{2}\left(\Omega ; \mathbb{R}^{3}\right)\right), \\
p^{\eta} & \in H^{1}\left(0, T ; H^{1}(\Omega ; \mathbb{R})\right), & \varepsilon^{p, \eta} & \in W^{1, \infty}\left(0, T ; L^{2}\left(\Omega ; \mathcal{S}^{3}\right)\right) .
\end{aligned}
$$

Proof. The energy estimate (Theorem 4.3) gives us the following information: The sequence $\left\{u^{\lambda}, \varepsilon^{p, \lambda}\right\}$ is bounded in $W^{1, \infty}\left(0, T ; H^{1}\left(\Omega ; \mathbb{R}^{3}\right) \times L^{2}\left(\Omega ; \mathcal{S}^{3}\right)\right)$, the sequence $u_{t t}^{\lambda}$ is bounded in $L^{\infty}\left(0, T ; L^{2}\left(\Omega ; \mathbb{R}^{3}\right)\right)$ and the sequence $\left\{p^{\lambda}\right\}$ is bounded in $H^{1}\left(0, T ; H^{1}(\Omega ; \mathbb{R})\right)$. Hence in the first four equations of the system (4.5) we can easily pass to the limit when $\lambda$ tends to $0^{+}$. Passing to the limit in the Lipschitz nonlinearities is the main difficult in the proof of this theorem.

From Theorem 4.4 we conclude that the sequence $\left\{\widehat{T}^{\eta, \lambda}\right\}$ is a Cauchy sequence in the space $L^{\infty}\left(0, T ; L^{2}\left(\Omega ; \mathcal{S}^{3}\right)\right)$ and from the definition of the Yosida approximation we get

$$
g^{\eta, \lambda}\left(\widehat{T}^{\eta, \lambda}\right)=g^{\eta}\left(J_{\lambda}\left(\widehat{T}^{\eta, \lambda}\right)\right) .
$$

We know that the sequence $\left(J_{\lambda}\left(\widehat{T}^{\eta, \lambda}\right), g^{\eta, \lambda}\left(\widehat{T}^{\eta, \lambda}\right)\right)$ is contained in the graph of $g^{\eta}$ and converges strongly-weakly to $\left(\widehat{T}^{\eta}, g^{\eta}\right)\left(J_{\lambda}\right.$ is a globally Lipshitz operator). From the strong-weak closedness of the graph of the maximal monotone operator $g^{\eta}$ we have

$$
\mathrm{w}-\lim _{\lambda \rightarrow 0^{+}} g^{\eta, \lambda}\left(\widehat{T}^{\eta, \lambda}\right)=g^{\eta}\left(\widehat{T}^{\eta}\right) .
$$

The properties of $G^{\eta}$ ( $G^{\eta}$ can be written as the sum of maximal monotone operator and a Lipschitz operator) implies that

$$
\mathrm{w}-\lim _{\lambda \rightarrow 0^{+}} G^{\eta, \lambda}\left(\widehat{T}^{\eta, \lambda}\right)=G^{\eta}\left(\widehat{T}^{\eta}\right)
$$

(for the properties of the Yosida approximation the reader may consult [4]).

This finishes the proof of the existence solution for the system (4.1), because the energy estimate yields that the sequence $\left\{G^{\lambda}\left(\widehat{T}^{\lambda}\right)\right\}$ is bounded in $L^{\infty}\left(0, T ; L^{2}\left(\Omega ; \mathcal{S}^{3}\right)\right)$.

The uniqueness follows immediately from coerciveness of the energy function evaluated on the difference of two solutions of the system (4.1).

\section{Proof of the Theorem 2.3}

This section is the main part of the proof of Theorem 2.3. We are going to prove some bounds on the approximate solutions of the system (1.1) and their derivatives. First, we formulate the definition of the weak safe load condition, which is known in the theory of inelastic deformation processes and it is only a condition on the boundary data (see for example [7]). 
Definition 5.1. We say that the given data $g_{d}$ and $g_{n}$ satisfy the weak safe load condition if there exist functions $\widetilde{u}^{0}$ and $\widetilde{u}^{1}$ such that the unique solution $(\widetilde{u}, \widetilde{T})$ of the linear system

$$
\begin{aligned}
\rho \widetilde{u}_{t t}(x, t)-\operatorname{div}_{x} \widetilde{T}(x, t) & =0, \\
\widetilde{T}(x, t) & =\mathcal{D}(\varepsilon(\widetilde{u}(x, t))), \\
\left.\widetilde{u}(x, t)\right|_{\Gamma_{d}} & =\left.g_{d}(x, t)\right|_{\Gamma_{d}},\left.\quad \widetilde{T}(x, t) n(x)\right|_{\Gamma_{n}}=\left.g_{n}(x, t)\right|_{\Gamma_{n}},
\end{aligned}
$$

with the initial conditions

$$
\left(\widetilde{u}(0), \widetilde{u}_{t}(0)\right)=\left(\widetilde{u}^{0}, \widetilde{u}^{1}\right)
$$

compatible with the boundary data, has the following regularity: for all $T>0$

$$
\begin{aligned}
\widetilde{u} & \in W^{1, \infty}\left((0, T) ; H^{1}\left(\Omega ; \mathbb{R}^{3}\right)\right), \\
\widetilde{T} & \in L^{\beta+1}\left((0, T) ; L^{\beta+1}\left(\Omega ; \mathcal{S}^{3}\right)\right), \\
\widetilde{u}_{t t} & \in L^{\infty}\left(0, T ; L^{2}\left(\Omega ; \mathcal{S}^{3}\right)\right) .
\end{aligned}
$$

Now we are ready to prove some estimates for the approximation sequence.

THEOREM 5.2 (Energy estimate). Suppose that our data have regularity required in (2.1)-(2.5). Let $\beta>r^{\prime}>r=\alpha \operatorname{deg}(Y)(\operatorname{deg}(Y)-1)>1$. Then there exists a positive constant $C(T)$ (not depending on $\eta$ ) such that the following inequality holds:

$$
\begin{aligned}
& \mathcal{E}^{\eta}\left(u_{t}^{\eta}-\widetilde{u}_{t}, \varepsilon^{\eta}-\widetilde{\varepsilon}, \varepsilon^{p, \eta}\right)(t)+\eta \int_{0}^{t} \int_{\Omega}\left|\widehat{T}^{\eta}\right|^{\beta+1} d x d \tau \\
& +\int_{0}^{t} \int_{\Omega} \mathcal{F}\left(Y\left(\widehat{T}^{\eta}\right)\right) \frac{\partial P}{\partial T}\left(\widehat{T}^{\eta}\right) \widehat{T}^{\eta} d x d \tau+c \int_{0}^{t} \int_{\Omega}\left|\nabla\left(p^{\eta}-p^{*}\right)\right|^{2} d x d \tau \leq C(T),
\end{aligned}
$$

where $p^{*} \in W^{2, \infty}\left(0, T ; H^{2}(\Omega ; \mathbb{R})\right)$ and $\left.p^{*}\right|_{\Gamma_{p}}=g_{\left.p\right|_{\Gamma_{p}}}$.

Proof. Calculate the time derivative of the energy to obtain

$$
\begin{aligned}
\frac{d}{d t}( & \left(\mathcal{E}^{\eta}\left(u_{t}^{\eta}-\widetilde{u}_{t}, \varepsilon^{\eta}-\widetilde{\varepsilon}, \varepsilon^{p, \eta}\right)(t)\right)=\rho \int_{\Omega}\left(u_{t}^{\eta}-\widetilde{u}_{t}\right)\left(u_{t t}^{\eta}-\widetilde{u}_{t t}\right) d x \\
& +\int_{\Omega} \mathcal{D}\left(\varepsilon^{\eta}-\widetilde{\varepsilon}-\varepsilon^{p, \eta}\right)\left(\varepsilon_{t}^{\eta}-\widetilde{\varepsilon}_{t}-\varepsilon_{t}^{p, \eta}\right) d x+\eta \int_{\Omega} \mathcal{D}\left(\varepsilon^{\eta}-\widetilde{\varepsilon}\right)\left(\varepsilon_{t}^{\eta}-\widetilde{\varepsilon}_{t}\right) d x \\
= & \rho \int_{\Omega}\left(u_{t}^{\eta}-\widetilde{u}_{t}\right)\left(u_{t t}^{\eta}-\widetilde{u}_{t t}\right) d x \\
& +\int_{\Omega}\left(T^{\eta}-(1+\eta) \widetilde{T}\right)\left(\nabla u_{t}^{\eta}-\nabla \widetilde{u}_{t}\right) d x-\int_{\Omega}\left(\widehat{T}^{\eta}-\widetilde{T}\right) \varepsilon_{t}^{p, \eta} d x \\
= & \rho \int_{\Omega}\left(u_{t}^{\eta}-\widetilde{u}_{t}\right)\left(u_{t t}^{\eta}-\widetilde{u}_{t t}\right) d x-\int_{\Omega} \operatorname{div}\left(T^{\eta}-(1+\eta) \widetilde{T}\right)\left(u_{t}^{\eta}-\widetilde{u}_{t}\right) d x \\
& +\int_{\partial \Omega}\left(T^{\eta}-(1+\eta) \widetilde{T}\right) n\left(u_{t}^{\eta}-\widetilde{u}_{t}\right) d S(x)-\int_{\Omega}\left(\widehat{T}^{\eta}-\widetilde{T}\right) \varepsilon_{t}^{p, \eta} d x .
\end{aligned}
$$


Using the first two equations from (4.1) and the weak safe load condition we get

$$
\begin{aligned}
\frac{d}{d t}\left(\mathcal{E}^{\eta}\left(u_{t}^{\eta}-\widetilde{u}_{t}, \varepsilon^{\eta}-\widetilde{\varepsilon}, \varepsilon^{p, \eta}\right)(t)\right)=\int_{\Omega}\left(F-\nabla p^{*}\right)\left(u_{t}^{\eta}-\widetilde{u}_{t}\right) d x \\
\quad+\eta \int_{\Omega} \operatorname{div} \widetilde{T}\left(u_{t}^{\eta}-\widetilde{u}_{t}\right) d x-\int_{\Omega}\left(\nabla p^{\eta}-\nabla p^{*}\right)\left(u_{t}^{\eta}-\widetilde{u}_{t}\right) d x \\
\quad+\int_{\partial \Omega}\left(T^{\eta}-(1+\eta) \widetilde{T}\right) n\left(u_{t}^{\eta}-\widetilde{u}_{t}\right) d S(x)-\int_{\Omega}\left(\widehat{T}^{\eta}-\widetilde{T}\right) \varepsilon_{t}^{p, \eta} d x \\
=\int_{\Omega}\left(F-\nabla p^{*}\right)\left(u_{t}^{\eta}-\widetilde{u}_{t}\right) d x \\
\quad+\eta \int_{\Omega} \operatorname{div} \widetilde{T}\left(u_{t}^{\eta}-\widetilde{u}_{t}\right) d x-\eta \int_{\Gamma_{n}} \widetilde{T} n\left(u_{t}^{\eta}-\widetilde{u}_{t}\right) d S(x) \\
\quad+\int_{\Omega} \operatorname{div}\left(u_{t}^{\eta}-\widetilde{u}_{t}\right)\left(p^{\eta}-p^{*}\right) d x-\int_{\Omega}\left(\widehat{T}^{\eta}-\widetilde{T}^{\prime}\right) \varepsilon_{t}^{p, \eta} d x \\
=\int_{\Omega}\left(F-\nabla p^{*}\right)\left(u_{t}^{\eta}-\widetilde{u}_{t}\right) d x \\
\quad+\eta \int_{\Omega} \operatorname{div} \widetilde{T}\left(u_{t}^{\eta}-\widetilde{u}_{t}\right) d x-\eta \int_{\Gamma_{n}} g_{n}\left(u_{t}^{\eta}-\widetilde{u}_{t}\right) d S \\
\quad-\int_{\Omega} \operatorname{div} \widetilde{u}_{t}\left(p^{\eta}-p^{*}\right) d x-c \int_{\Omega}\left|\nabla p^{\eta}-\nabla p^{*}\right|^{2} d x \\
\quad-\int_{\Omega}\left(f-c \Delta p^{*}\right)\left(p^{\eta}-p^{*}\right) d x+\int_{\Gamma_{v}} g_{v}\left(p^{\eta}-p^{*}\right) d S(x) \\
\quad-c \int_{\Gamma_{v}}\left(p^{\eta}-p^{*}\right) \frac{\partial p^{*}}{\partial n} d S(x)-\int_{\Omega}\left(\widehat{T}^{\eta}-\widetilde{T}\right) \varepsilon_{t}^{p, \eta} d x .
\end{aligned}
$$

Integrating the whole equality with respect to time we obtain

$$
\begin{aligned}
& \mathcal{E}^{\eta}\left(u_{t}^{\eta}-\widetilde{u}_{t}, \varepsilon^{\eta}-\widetilde{\varepsilon}, \varepsilon^{p, \eta}\right)(t)+\eta \int_{0}^{t} \int_{\Omega}\left|\widehat{T}^{\eta}\right|^{\beta+1} d x d \tau \\
& +\int_{0}^{t} \int_{\Omega} \mathcal{F}\left(Y\left(\widehat{T}^{\eta}\right)\right) \frac{\partial P}{\partial T}\left(\widehat{T}^{\eta}\right) \widehat{T}^{\eta} d x d \tau+c \int_{0}^{t} \int_{\Omega}\left|\nabla p^{\eta}-\nabla p^{*}\right|^{2} d x d \tau \\
& =\mathcal{E}^{\eta}\left(u_{t}^{\eta}-\widetilde{u}_{t}, \varepsilon^{\eta}-\widetilde{\varepsilon}, \varepsilon^{p, \eta}\right)(0) \\
& +\int_{0}^{t} \int_{\Omega}\left(F-\nabla p^{*}\right)\left(u_{t}^{\eta}-\widetilde{u}_{t}\right) d x d \tau+\eta \int_{0}^{t} \int_{\Omega} \operatorname{div} \widetilde{T}\left(u_{t}^{\eta}-\widetilde{u}_{t}\right) d x d \tau \\
& -\eta \int_{0}^{t} \int_{\Gamma_{n}} g_{n}\left(u_{t}^{\eta}-\widetilde{u}_{t}\right) d S(x) d \tau-\int_{0}^{t} \int_{\Omega} \operatorname{div} \widetilde{u}_{t}\left(p^{\eta}-p^{*}\right) d x d \tau \\
& -\int_{0}^{t} \int_{\Omega}\left(p^{\eta}-p^{*}\right)\left(f-c \Delta p^{*}\right) d x d \tau+\int_{0}^{t} \int_{\Gamma_{v}} g_{v}\left(p^{\eta}-p^{*}\right) d S(x) d \tau \\
& -c \int_{0}^{t} \int_{\Gamma_{v}}\left(p^{\eta}-p^{*}\right) \frac{\partial p^{*}}{\partial n} d S(x) d \tau+\int_{0}^{t} \int_{\Omega} \widetilde{T} \varepsilon_{t}^{p, \eta} d x d \tau .
\end{aligned}
$$

From the assumption on the initial data, the first term on the right-hand side of (5.4) is bounded independently of $\eta$. 
Now we estimate the following integral:

$$
\begin{array}{r}
-\eta \int_{0}^{t} \int_{\Gamma_{n}} g_{n}\left(u_{t}^{\eta}-\widetilde{u}_{t}\right) d S(x) d \tau=\eta \int_{0}^{t} \int_{\Gamma_{n}} \partial_{t} g_{n}\left(u^{\eta}-\widetilde{u}\right) d S(x) d \tau \\
-\eta \int_{\Gamma_{n}} g_{n}\left(u^{\eta}-\widetilde{u}\right) d S+\eta \int_{\Gamma_{n}} g_{n}(0)\left(u^{\eta}(0)-\widetilde{u}(0)\right) d S(x) .
\end{array}
$$

Using the regularity of $g_{n}(0), u^{\eta}(0)$ and $\widetilde{u}$ we note that the last integral in (5.5) is bounded. Moreover, $\left.\left(u^{\eta}-\widetilde{u}\right)\right|_{\Gamma_{d}}=0$ so, from the Korn's inequality $\left\|u^{\eta}-\widetilde{u}\right\|_{H^{1}\left(\Omega ; \mathbb{R}^{3}\right)} \leq C(\Omega)\left\|\varepsilon\left(u^{\eta}-\widetilde{u}\right)\right\|_{L^{2}\left(\Omega ; \mathcal{S}^{3}\right)}$. Using the continuity of the trace operator we arrive at the inequality

$$
\begin{aligned}
& \text { 6) } \quad-\eta \int_{0}^{t} \int_{\Gamma_{n}} g_{n}\left(u_{t}^{\eta}-\widetilde{u}_{t}\right) d S d \tau \leq \frac{1}{2} \int_{0}^{t}\left\|\partial_{t} g_{n}\right\|_{L^{2}\left(\Gamma_{n} ; \mathbb{R}^{3}\right)}^{2} d \tau \\
& +c \int_{0}^{t} \eta\left\|\varepsilon^{\eta}-\widetilde{\varepsilon}\right\|_{L^{2}\left(\Omega ; \mathcal{S}^{3}\right)}^{2} d \tau+C(\nu)\left\|g_{n}\right\|_{L^{2}\left(\Gamma_{n} ; \mathbb{R}^{3}\right)}+\nu \eta\left\|\varepsilon^{\eta}-\widetilde{\varepsilon}\right\|_{L^{2}\left(\Omega ; \mathcal{S}^{3}\right)}+C
\end{aligned}
$$

where $\nu>0$ is any positive number. Next, by Young's inequality we obtain

$$
\begin{aligned}
\int_{0}^{t} \int_{\Omega} \widetilde{T} \varepsilon_{t}^{p, \eta} d x d \tau \leq C(\Omega, \beta, \nu) \int_{0}^{t}\|\widetilde{T}\|_{L^{\beta+1}\left(\Omega ; \mathcal{S}^{3}\right)}^{\beta+1} d \tau & \\
& \quad+\nu \int_{0}^{t}\left\|\varepsilon_{t}^{p, \eta}\right\|_{L^{1+1 / \beta}\left(\Omega ; \mathcal{S}^{3}\right)}^{1+1 / \beta} d \tau
\end{aligned}
$$

From the last equation in (4.1) we have (Observe that $1+1 / \beta<1+1 / r$ )

$$
\begin{aligned}
\int_{0}^{t}\left\|\varepsilon_{t}^{p, \eta}\right\|_{L^{1+1 / \beta}\left(\Omega ; \mathcal{S}^{3}\right)}^{1+1 / \beta} d \tau=\int_{0}^{t} \int_{\Omega}\left|\varepsilon_{t}^{p, \eta}\right|^{1+1 / \beta} d x d \tau \\
\leq 2^{1 / \beta}\left(\left.\left.\int_{0}^{t} \int_{\Omega}|\eta| \widehat{T}^{\eta}(x, t)\right|^{\beta}\right|^{1+1 / \beta} d x d \tau\right. \\
\left.\quad+\int_{0}^{t} \int_{\Omega}\left|\mathcal{F}\left(Y\left(\widehat{T}^{\eta}(x, t)\right)\right) \frac{\partial P}{\partial T}\left(\widehat{T}^{\eta}(x, t)\right)\right|^{1+1 / \beta} d x d \tau\right)
\end{aligned}
$$

$\left(\eta^{1+1 / \beta}<\eta\right.$ for $\left.\eta<1\right)$

$$
\begin{aligned}
\leq & C(\beta) \eta \int_{0}^{t}\left\|\widehat{T}^{\eta}\right\|_{L^{\beta+1}\left(\Omega ; \mathcal{S}^{3}\right)}^{\beta+1} d \tau \\
& +C(\Omega, \beta, r) \int_{0}^{t}\left\|\mathcal{F}\left(Y\left(\widehat{T}^{\eta}\right)\right) \frac{\partial P}{\partial T}\left(\widehat{T}^{\eta}\right)\right\|_{L^{1+1 / r}\left(\Omega ; \mathcal{S}^{3}\right)}^{1+1 / r} d \tau .
\end{aligned}
$$

Other terms on the right-hand side of (5.4) are bounded by standard methods: continuity of the trace operator and Cauchy inequality (see for instance [17]). If 
we use the inequalities (5.5)-(5.8) then we obtain that the inequality

$$
\begin{aligned}
& \mathcal{E}^{\eta}\left(u_{t}^{\eta}-\widetilde{u}_{t}, \varepsilon^{\eta}-\widetilde{\varepsilon}, \varepsilon^{p, \eta}\right)(t)+\eta \int_{0}^{t} \int_{\Omega}\left|\widehat{T}^{\eta}\right|^{\beta+1} d x d \tau \\
& \quad+\int_{0}^{t} \int_{\Omega} \mathcal{F}\left(Y\left(\widehat{T}^{\eta}\right)\right) \frac{\partial P}{\partial T}\left(\widehat{T}^{\eta}\right) \widehat{T}^{\eta} d x d \tau+c \int_{0}^{t} \int_{\Omega}\left|\nabla\left(p^{\eta}-p^{*}\right)\right|^{2} d x d \tau \\
& \leq C(\Omega, \beta, T, \nu)+\nu \sup _{t \in(0, T)}\left\|u_{t}^{\eta}-\widetilde{u}_{t}\right\|_{L^{2}\left(\Omega ; \mathbb{R}^{3}\right)}^{2} \\
& \quad+\sup _{t \in(0, T)} \nu \eta\left\|\varepsilon^{\eta}-\widetilde{\varepsilon}\right\|_{L^{2}\left(\Omega ; \mathcal{S}^{3}\right)}+\nu C(\Omega) c \int_{0}^{t} \int_{\Omega}\left|\nabla\left(p^{\eta}-p^{*}\right)\right|^{2} d x d \tau \\
& \quad+\nu C(\beta) \eta \int_{0}^{t}\left\|\widehat{T}^{\eta}\right\|_{L^{\beta+1}\left(\Omega ; \mathcal{S}^{3}\right)}^{\beta+1} d \tau \\
& \quad+C(\Omega, \beta, r) \nu \int_{0}^{t}\left\|\mathcal{F}\left(Y\left(\widehat{T}^{\eta}\right)\right) \frac{\partial P}{\partial T}\left(\widehat{T}^{\eta}\right)\right\|_{L^{1+1 / r}\left(\Omega ; \mathcal{S}^{3}\right)}^{1+1 / r} d \tau
\end{aligned}
$$

holds for any $\nu>0$ and the constant $C(\Omega, \beta, T, \nu)>0$ does not depend on $\eta>0$. The inequality (5.9) is similar to the inequality from the proof of Theorem 4.1 in [18]. Doing a proof analogous to that one we have finished.

Note that to pass to the limit in the system (4.1) with $\eta \rightarrow 0^{+}$(in the sense of the Definition 2.1) we have to bound the expression $\int_{0}^{T}\left\|u_{t t}^{\eta}\right\|_{V^{\star}}^{2} d \tau$. Fix $v \in V$ which satisfies $\|v\|_{V} \leq 1$. Using Theorem 4.5 we obtain the following equation

$$
\begin{aligned}
\rho \int_{\Omega} u_{t t}^{\eta} v d x+\int_{\Omega} \mathcal{D}\left(\varepsilon\left(u^{\eta}\right)-\varepsilon^{p, \eta}+\eta \varepsilon\left(u^{\eta}\right)\right) \varepsilon(v) & d x-\int_{\Omega} p^{\eta} \operatorname{div} v d x \\
& =\int_{\Omega} F v d x+\int_{\Gamma_{n}} g_{n} v d S(x) .
\end{aligned}
$$

Therefore

$$
\begin{aligned}
\rho\left\langle u_{t t}^{\eta}, v\right\rangle=\rho \int_{\Omega} u_{t t}^{\eta} v d x & =\int_{\Omega} F v d x+\int_{\Gamma_{n}} g_{n} v d S \\
& -\int_{\Omega} \mathcal{D}\left(\varepsilon\left(u^{\eta}\right)-\varepsilon^{p, \eta}+\eta \varepsilon\left(u^{\eta}\right)\right) \varepsilon(v) d x+\int_{\Omega} p^{\eta} \operatorname{div} v d x
\end{aligned}
$$

Using $\|v\|_{V} \leq 1$ and Theorem 5.2 we have

$$
\rho \int_{0}^{T}\left\|u_{t t}^{\eta}\right\|_{V^{\star}}^{2} d \tau \leq C(T) .
$$

Proof of Theorem 2.3. The energy estimate proved in the last theorem yields that the sequence $\left\{\widehat{T}^{\eta}, \sqrt{\eta} \varepsilon^{\eta}\right\}_{\eta>0}$ is bounded in $L^{\infty}\left(0, T ; L^{2}\left(\Omega ; \mathcal{S}^{3}\right) \times\right.$ $\left.L^{2}\left(\Omega ; \mathcal{S}^{3}\right)\right),\left\{p^{\eta}\right\}_{\eta>0}$ is bounded in $L^{2}\left(0, T ; H^{1}(\Omega ; \mathbb{R})\right)$ and $\left\{\varepsilon^{p, \eta}\right\}_{\eta>0}$ is bounded in $W^{1,1+1 / \beta}\left(0, T ; L^{1+1 / \beta}\left(\Omega ; \mathcal{S}^{3}\right)\right)$. Using this information we also obtain that $\left\{\varepsilon^{\eta}\right\}_{\eta>0}$ is bounded in $L^{1+1 / \beta}\left(0, T ; L^{1+1 / \beta}\left(\Omega ; \mathcal{S}^{3}\right)\right),\left\{\operatorname{div} u^{\eta}=\operatorname{tr}\left(\varepsilon\left(u^{\eta}\right)\right)\right\}_{\eta>0}$ is bounded in $L^{1+1 / \beta}\left(0, T ; L^{1+1 / \beta}(\Omega ; \mathbb{R})\right), u_{t t}^{\eta}$ is bounded in $L^{2}\left(0, T ;\left(H^{1}\left(\Omega ; \mathbb{R}^{3}\right)\right)^{*}\right)$. 
Notice that this information is enough to pass to the limit in the first four equations of the system (4.1) (for analogous calculations we refer to [9]). It is easy to see that $\left\{\varepsilon_{t}^{p, \eta}\right\}_{\eta>0}$ is weakly precompact in $L^{1}\left(0, T ; L^{1}\left(\Omega ; \mathcal{S}^{3}\right)\right)$ and the expression $\eta\left|\widehat{T}^{\eta}\right|^{\beta} \widehat{T}^{\eta} /\left|\widehat{T}^{\eta}\right|$ tends to zero in $L^{1}\left(0, T ; L^{1}\left(\Omega ; \mathcal{S}^{3}\right)\right)$ as $\eta \rightarrow 0^{+}$. The sequence

$$
\left\{\mathcal{F}\left(Y\left(\widehat{T}^{\eta}\right)\right) \frac{\partial P}{\partial T}\left(\widehat{T}^{\eta}\right)\right\}_{\eta>0}
$$

is weakly precompact in $L^{1}\left(0, T ; L^{1}\left(\Omega ; \mathcal{S}^{3}\right)\right)$. Then, there exists a family of Young measures $\nu_{(x, t)}$ (see [5]) generated by the sequence $\left\{\widehat{T}^{\eta}\right\}_{\eta>0}$ such that $w-\lim _{\eta \rightarrow 0^{+}} \varepsilon_{t}^{p, \eta}=\widehat{\chi}$ is in the form

$$
\widehat{\chi}(x, t)=\int_{\mathcal{S}^{3}} \mathcal{F}(Y(S)) \frac{\partial P}{\partial T}(S) d \nu_{(x, t)}(S) .
$$

Acknowledgements. This work has been partially supported by the Polish Ministry of Science and Higher Education Grant No. N N201 392537 and partially supported by the European Union in the framework of the European Social Fund through the Warsaw University of Technology Development Programme. The author would like to thank K. Chełmiński for his constant support.

\section{REFERENCES}

[1] R.A. Adams And J.J.F. Fournier, Sobolev Spaces, Second edition, Pure and Applied Mathematics (Amsterdam), vol. 140, Elsevier/Academic Press, Amsterdam, 2003.

[2] H.-D. Alber, Materials with memory, Lecture Notes in Math., vol. 1682, Springer, Berlin, Heidelberg, New York, 1998.

[3] H.-D. Alber and K. Chęmiński, Quasistatic problems in viscoplasticity theory I: Models with linear hardening, Operator Theoretical Methods and Applications to Mathematical Physics (I. Gohberg et al., eds.), The Erhard Meister memorial volume, Birkhäuser, Basel, 2004, pp. 105-129.

[4] J.P. Aubin and A. Cellina, Differential Inclusions, Springer, Berlin, Heidelberg, New York, 1984.

[5] J.M. BALL, A version of the fundamental theorem for Young measures, PDEs and continuum models of phase transitions (Nice, 1988),, Lecture Notes in Phys., vol. 334 Springer, Berlin, 1989, pp. 207-215.

[6] K. CheŁmiński, Coercive limits for a subclass of monotone constitutive equations in the theory of inelastic material behaviour of metals, Roczniki PTM: App. Math. 40 (1997), 41-81.

[7] Coercive approximation of viscoplasticity and plasticity, Asymptot. Anal. 26 (2001), 115-135.

[8] K. Chemmiński and P. Gwiazda, Monotonicity of operators of viscoplastic response: application to the model of Bodner-Partom, Bull. Pol. Acad. Sci. Tech. Sci. 47 (1999), $191-208$

[9] Nonhomogeneous initial-boundary value problems for coercive and self-controlling models of monotone type, Contin. Mech. Thermodyn. 12 (2000), 217-234. 
[10] K. Chemmiński And P. GWiazda, Convergence of coercive approximations for strictly monotone quasistatic models in deformation teory, Math. Methods Appl. Sci. 30 (2007), $1357-1374$.

[11] R.J. DiPERnA AND A. MAJDA, Reduced Hausdorff dimension and concentration-cancellation for two-dimensional incompressible flow, J. Amer. Math. Soc. 1 (1988), 59-95.

[12] W. EHLERs, Constitutive equations for granular materials in geomechanical context, Continuum Mechanics in Environmental Sciences and Geophysics (K. Hutter, ed.), CISM Courses and Lectures, vol. 337, Springer-Verlag, Wien, 1993, pp. 313-402.

[13] P. GWIAZDA, On measure-valued solutions to a two-dimensional gravity-driven avalanche flow model, Math. Methods Appl. Sci. 28 (2005), 2201-2223.

[14] P. Gwiazda, J. Málek And A. Świerczewska, On flows of an incompressible fluid with a discontinuous power-law-like rheology, Comput. Math. Appl. 53 (2007), 531-546.

[15] J. MÁLEK, J. NEČAs, M. ROKYTA AND M. RøUĂIČKA, Weak and measure-valued solutions to evolutionary PDEs, Applied Mathematics and Mathematical Computation, vol. 13, Chapman and Hall, London, 1996.

[16] S. Owczarek, A Galerkin method for Biot consolidation model, Math. Mech. Solids 15 (2010), 42-56.

[17] Convergence of coercive approximations for a model of gradient type in poroplasticity, Math. Methods Appl. Sci. 32 (2009), 1541-1563.

[18] Convergence of a monotonisation procedure for a non-monotone quasi-static model in poroplasticity, J. Math. Anal. Appl. 364 (2010), 599-608.

[19] R.E. Showalter, Diffusion in poro-elastic media, J. Math. Appl. 251 (2000), 310-340.

[20] R. Temam, Problèmes mathématiques en plasticité, Bordas, Paris, 1983.

Sebastian OwCZAREK

Faculty of Mathematics and Information Science

Warsaw University of Technology

Koszykowa 75

00-662 Warszawa, POLAND

E-mail address: s.owczarek@mini.pw.edu.pl 